\title{
A Fuzzy Logic Based Resolution Principal for Approximate Reasoning
}

\author{
Alex Tserkovny \\ Dassault Systemes, Boston, MA, USA \\ Email: atserkovny@yahoo.com
}

How to cite this paper: Tserkovny, A. (2017) A Fuzzy Logic Based Resolution Principal for Approximate Reasoning. Journal of Software Engineering and Applications, 10, 793-823. https://doi.org/10.4236/jsea.2017.1010045

Received: August 26, 2017

Accepted: September 25, 2017

Published: September 28, 2017

Copyright $\odot 2017$ by author and Scientific Research Publishing Inc. This work is licensed under the Creative Commons Attribution International License (CC BY 4.0).

http://creativecommons.org/licenses/by/4.0/

\section{(c) (i) Open Access}

\begin{abstract}
In this article, we present a systemic approach toward a fuzzy logic based formalization of an approximate reasoning methodology in a fuzzy resolution, where we derive a truth value of $A$ from both values of $B \rightarrow A$ and $B$ by some mechanism. For this purpose, we utilize a t-norm fuzzy logic, in which an implication operator is a root of both graduated conjunction and disjunction operators. Furthermore by using an inverse approximate reasoning, we conclude the truth value of $\mathrm{A}$ from both values of $\mathrm{B} \rightarrow \mathrm{A}$ and $\mathrm{B}$, applying an altogether different mechanism. A current research is utilizing an approximate reasoning methodology, which is based on a similarity relation for a fuzzification, while similarity measure is utilized in fuzzy inference mechanism. This approach is applied to both generalized modus-ponens/modus-tollens syllogisms and is well-illustrated with artificial examples.
\end{abstract}

\section{Keywords}

Fuzzy Logic, Deduction, Fuzzy Resolvent, Implication, Disjunction, Conjunction, Antecedent, Consequent, Modus-Ponens, Modus-Tollens, Fuzzy Conditional Inference Rule

\section{Introduction}

This study is a continuation of a research, which is based on a proposed t-norm fuzzy logic, presented in [1]. Here we also use an automated theorem proving, where a resolution principal is a rule of an inference, leading to a refutation theorem-proving technique. Applying the resolution rule in a suitable way, it is possible to check whether a propositional formula is Universally Valid (UV) and construct a proof of a fact that relative consequent's first-order formula is $U V$ or non $U V$. In 1965, J. A. Robinson [2] introduced the resolution principle for first-order logic. A fuzzy resolution principal, in its part, was introduced by $\mathrm{M}$. 
Mukaidono [3].

Taking into account the above mentioned, we present the following.

Definition 1 [3].

A fuzzy resolvent of two fuzzy clauses $\widetilde{C_{1}}$ and $\widetilde{C_{2}}$, containing the complementary literals $x_{i}$ and $\neg x_{i}$ respectively, is defined as

$$
R\left(\widetilde{C_{1}}, \widetilde{C_{2}}\right)=L_{1} \vee L_{2}
$$

where $\widetilde{C_{1}}=x_{i} \vee L_{1}$ and $\widetilde{C_{2}}=\neg x_{i} \vee L_{2} . L_{1}, L_{2}$ are fuzzy clauses, which don't contain $x_{i}$ and $\neg x_{i}$ respectively. The operator $\vee$ is understood as the disjunction of the literals present in them. It is also a logical consequence of $\widetilde{C_{1}} \wedge \widetilde{C_{2}}$. A resolution deduction of a clause $\widetilde{C}$ from a set $S$ of clauses is a finite sequence of clauses $\widetilde{C_{1}}, \widetilde{C_{2}}, \cdots, \widetilde{C_{n}}=\widetilde{C}$ such that each $\widetilde{C}_{i}$ is either a member of or is a resolvent of two clauses taken from the resolution principle in propositional logic we deduce that, if $S$ is true under some truth valuation $v$, then $v\left(C_{i}\right)=$ TRUE for all $i$, and in particular, $v(C)=$ TRUE [2].

Example 1: Here is a derivation of a clause from a set of clauses presented by means of a resolution Tree in Figure 1.

In first order logic, resolution condenses the traditional syllogism of logical inference down to single rule.

A simple resolution scheme is:

Antecedent 1: $a \vee b$

Antecedent 2: $\neg b$

Consequent: $a$.

The entire historical analysis of this approach toward applying of a resolution principal to a logical inference is presented in [4].

\section{Basic Theoretical Aspects}

First, Let us consider that $A, A^{\prime}, B$ and $B^{\prime}$ are fuzzy concepts represented by fuzzy sets in universe of discourse $U, U, V$ and $V$, respectively and correspondent fuzzy sets be represented as such $A \subset U\left|\mu_{A}: U \rightarrow[0,1], B \subset V\right| \mu_{B}: V \rightarrow[0,1]$, where

$$
A=\int_{U} \mu_{A}(u) / u, \quad B=\int_{V} \mu_{B}(v) / v
$$

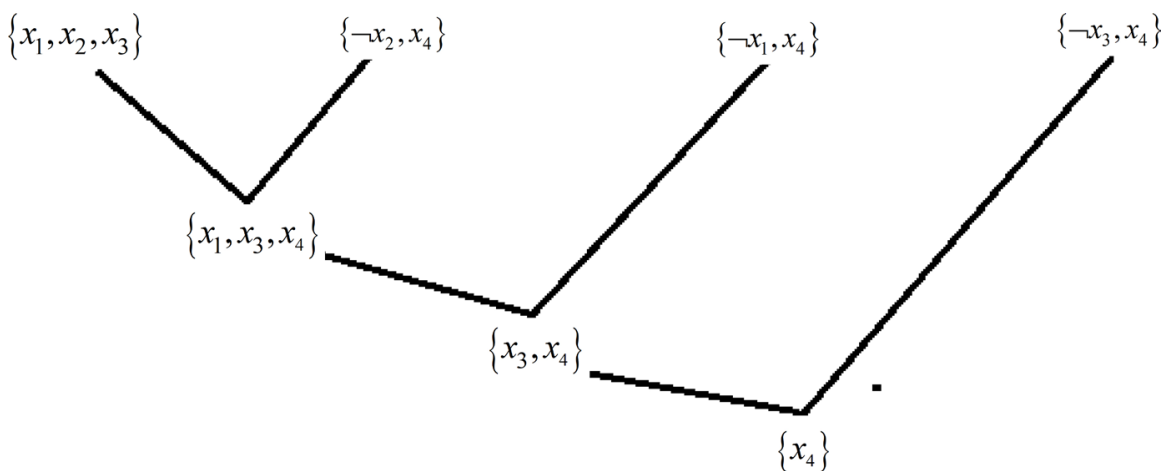

Figure 1. Resolution Tree. 
Given (2.1) let us formulate the argument form of simple Fuzzy Resolution as follows.

$$
\begin{gathered}
A \vee B \\
\neg B \\
A
\end{gathered}
$$

Given (2.2) the scheme for Generalized Fuzzy Resolution looks like that

$$
\begin{gathered}
\text { Antecedent 1: If } x \text { is } A O R \text { is } B \\
\text { Antecedent 2: } y \text { is } B^{\prime} \\
\text { Consequent: } x \text { is } A \text { ! }
\end{gathered}
$$

In case (2.3), we can say that the Disjunctive Syllogism holds if $B^{\prime}$ is close to not $B$, whereas $A$ 'is close to $A$. The second approach is called Inverse Approximate Reasoning. Its scheme looks like that:

$$
\begin{aligned}
& \text { Antecedent 1: If } x \text { is } A \text {, then } y \text { is } B \\
& \text { Antecedent 2: } y \text { is } B^{\prime} \\
& \text { Consequent: } x \text { is } A^{\prime} \text {. }
\end{aligned}
$$

We shall transform the disjunction form of rule into fuzzy implication from fuzzy logic, introduced in [1], or fuzzy relation and apply the method of inverse approximate reasoning to get the required resolvent. However, in the case of complex set of clauses the method is not suitable. Hence, we investigate for another method of approximate reasoning based on similarity to get the fuzzy resolvent.

Let us consider Generalized Fuzzy Resolution first. The key operation used in this method is disjunction. The disjunction operation $\vee$ is presented in Table S1 and, being applied to above introduced fuzzy sets $A$ and $B$, looks like that [1]

$$
A \vee B=\left\{\begin{array}{l}
A \cdot B, A+B<1, \\
1, A+B \geq 1
\end{array}\right.
$$

Whereas correspondent conjunction operation $\wedge$ is also presented in Table S1 and looks like that [1]

$$
A \wedge B=\left\{\begin{array}{l}
A \cdot B, A+B>1, \\
0, A+B \leq 1
\end{array}\right.
$$

Taking into account (2.5) and (2.6) and the fact that $\neg A=1-A$, let us formulate the following

\section{Lemma 1.}

If there are two fuzzy clauses $\widetilde{C_{1}}, \widetilde{C_{2}}$ and $R\left(\widetilde{C_{1}}, \widetilde{C_{2}}\right)$ is a fuzzy resolvent of them with keyword $x_{i}$, then the following inequality holds

$$
T\left(C_{1} \wedge \widetilde{C_{2}}\right) \leq T\left(R\left(\widetilde{C_{1}}, \widetilde{C_{2}}\right)\right)
$$

where $T(x)$ is a truth value of an $x$.

Proof: Since $\widetilde{C_{1}}=x_{i} \vee L_{1}$ and $\widetilde{C_{2}}=\neg x_{i} \vee L_{2}$, where $L_{1}, L_{2} \neq 0$, then 


$$
\begin{aligned}
C_{1} \wedge \widetilde{C_{2}} & =\left(x_{i} \vee L_{1}\right) \wedge\left(\neg x_{i} \vee L_{2}\right) \\
& =\left(x_{i} \wedge \neg x_{i}\right) \vee\left(L_{1} \wedge \neg x_{i}\right) \vee\left(x_{i} \wedge L_{2}\right) \vee\left(L_{1} \wedge L_{2}\right)
\end{aligned}
$$

whereas from (1.1) $R\left(\widetilde{C_{1}}, \widetilde{C_{2}}\right)=L_{1} \vee L_{2}$. From (2.8) let define the following values of truth:

$$
T_{1}=\left(x_{i} \wedge \neg x_{i}\right)=\left\{\begin{array}{l}
x_{i} \cdot \neg x_{i}, x_{i}+\neg x_{i}>1, \\
0, x_{i}+\neg x_{i} \leq 1
\end{array}\right.
$$

Since $x_{i}+\neg x \equiv 1$, then from (2.9) we are getting that

$$
T_{1} \equiv 0
$$

In a meantime from the same (2.8) we have

$$
T_{2}=\left(L_{1} \wedge \neg x_{i}\right)=\left\{\begin{array}{l}
L_{1} \cdot \neg x_{i}, L_{1}>x_{i}, \\
0, L_{1} \leq x_{i}
\end{array}\right.
$$

From (2.11) let's take a note that

$$
\operatorname{Sup}\left(T_{2}\right) \equiv 1 \mid L_{1}=1, x_{i}=0
$$

Also from (2.11) let

$$
T_{3}=\left(x_{i} \wedge L_{2}\right)=\left\{\begin{array}{l}
x_{i} \cdot L_{2}, x_{i}+L_{2}>1, \\
0, x_{i}+L_{2} \leq 1
\end{array}\right.
$$

Continuing from (2.11) let

$$
T_{4}=\left(L_{1} \wedge L_{2}\right)=\left\{\begin{array}{l}
L_{1} \cdot L_{2}, L_{1}+L_{2}>1, \\
0, L_{1}+L_{2} \leq 1
\end{array}\right.
$$

And finally from (1.1) we have

$$
L_{1} \vee L_{2}=\left\{\begin{array}{l}
L_{1} \cdot L_{2}, L_{1}+L_{2}<1, \\
1, L_{1}+L_{2} \geq 1
\end{array}\right.
$$

Let's rewrite (2.8) in the following way

$$
T\left(C_{1} \wedge \widetilde{C_{2}}\right)=T_{1} \vee T_{2} \vee T_{3} \vee T_{4}
$$

From (2.16) given both (2.10) and (2.12) we have

$$
T_{12}=T_{1} \vee T_{2}=0 \vee T_{2}=\left\{\begin{array}{l}
0, T_{2}<1, \\
1, T_{2} \geq 1
\end{array}=\left\{\begin{array}{l}
0, T_{2}<1, \\
1, T_{2}=1
\end{array}\right.\right.
$$

Taking into account (2.12) finally we are getting

$$
T_{12}=\left\{\begin{array}{l}
0, T_{2}<1 \\
1, L_{1}=1, x_{i}=0
\end{array}\right.
$$

Furthermore from the same (2.16) let

$$
T_{34}=T_{3} \vee T_{4}=\left\{\begin{array}{l}
T_{3} \cdot T_{4}, T_{3}+T_{4}<1, \\
1, T_{3}+T_{4} \geq 1
\end{array}\right.
$$

Note that from (2.18) $x_{i}=0$, which means that from (2.13) $T_{3} \equiv 0$, therefore from (2.19) we have 


$$
T_{34}=\left\{\begin{array}{l}
0, T_{4}<1 \\
1, T_{4} \geq 1
\end{array}\right.
$$

But from (2.14) and (2.20) $T_{4}=1$, when $L_{1} \cdot L_{2}=1$ or equally

$$
L_{1}=L_{2}=1
$$

Finally from (2.16), given (2.18) and (2.20), we are getting the following

$$
T\left(C_{1} \wedge \widetilde{C}_{2}\right)=T_{12} \vee T_{34}=\left\{\begin{array}{l}
T_{12} \cdot T_{34}, T_{12}+T_{34}<1, \\
1, T_{12}+T_{34} \geq 1
\end{array}=\left\{\begin{array}{l}
0, T_{12}+T_{34}<1, \\
1, T_{12}+T_{34} \geq 1
\end{array}\right.\right.
$$

Taking into account (2.21) from (2.15) and (1.1)

$$
R\left(\widetilde{C_{1}}, \widetilde{C_{2}}\right)=L_{1} \vee L_{2} \equiv 1
$$

From (2.22) and (2.23) we are getting

$$
T\left(C_{1} \wedge \widetilde{C_{2}}\right) \leq T\left(R\left(\widetilde{C_{1}}, \widetilde{C_{2}}\right)\right) \text { (Q. E. D.). }
$$

\section{Corollary 1}

Let $\widetilde{C_{1}}, \widetilde{C_{2}}$ are two fuzzy clauses. If $T\left(R\left(\widetilde{C_{1}}, \widetilde{C_{2}}\right)\right)<0.25$, then the following is true:

$$
T\left(C_{1} \wedge \widetilde{C_{2}}\right)<T\left(R\left(\widetilde{C_{1}}, \widetilde{C_{2}}\right)\right)
$$

Proof: First note that for

$$
L_{1}, L_{2} \in[0,1] \mid L_{1}+L_{2}<1 \Rightarrow L_{1} \cdot L_{2}<0.25
$$

From (1.1) and (2.15), given (2.25) we are getting the following

$$
T\left(R\left(\widetilde{C}_{1}, \widetilde{C_{2}}\right)\right)=L_{1} \vee L_{2}=\left\{\begin{array}{l}
L_{1} \cdot L_{2}, L_{1}+L_{2}<1, \\
1, L_{1}+L_{2} \geq 1
\end{array}=\left\{\begin{array}{l}
0.25, L_{1}+L_{2}<1, \\
1, L_{1}+L_{2} \geq 1
\end{array}\right.\right.
$$

First from (2.14) we have the following

$$
T_{4} \equiv 0 \mid L_{1}+L_{2} \leq 1 \Rightarrow T_{34}=T_{3} \vee 0=\left\{\begin{array}{l}
0, T_{3}<1, \\
1, T_{3}=1
\end{array}\right.
$$

But from (2.13) we have the following $T_{3}=1 \mid x_{i}=L_{2}=1 \Rightarrow L_{1} \equiv 0$, but $L_{1}+L_{2}<1 \Rightarrow T_{3} \equiv 0$, therefore

$T_{34} \equiv 0 \Rightarrow T_{12} \vee T_{34} \equiv 0$, i.e. $<0.25$, in other words the following is true.

$$
T\left(C_{1} \wedge \widetilde{C_{2}}\right)<T\left(R\left(\widetilde{C_{1}}, \widetilde{C_{2}}\right)\right) \text { (Q. E. D.). }
$$

\section{Corollary 2}

Let $\widetilde{C_{1}}, \widetilde{C_{2}}$ are two fuzzy clauses. If $T\left(R\left(\widetilde{C_{1}}, \widetilde{C_{2}}\right)\right) \geq 0.25$, then the following is true:

$$
T\left(C_{1} \wedge \widetilde{C_{2}}\right)=T\left(R\left(\widetilde{C_{1}}, \widetilde{C_{2}}\right)\right)
$$

\section{Proof:}

From (2.22) we have $T\left(\left(C_{1} \wedge \widetilde{C}_{2}\right)=\left\{\begin{array}{l}0, T_{12}+T_{34}<1, \\ 1, T_{12}+T_{34} \geq 1\end{array}\right.\right.$, which means that $T\left(C_{1} \wedge \widetilde{C_{2}}\right) \geq 0.25 \Rightarrow 1$. From (2.14), (2.17) and (2.19) we are getting 
$T\left(C_{1} \wedge \widetilde{C_{2}}\right) \equiv 1 \Rightarrow L_{1}=\widetilde{L_{2}}=1$, but from $(2.26) \quad T\left(R\left(\widetilde{C_{1}}, \widetilde{C_{2}}\right)\right) \equiv 1 \mid L_{1}=L_{2}=1$ which means that $T\left(C_{1} \wedge \widetilde{C}_{2}\right)=T\left(R\left(\widetilde{C_{1}}, \widetilde{C_{2}}\right)\right)$ (Q. E. D.).

Based on above presented results let formulate the following

Theorem 1 (Deduction):

Let $\widetilde{A}_{1}, \widetilde{A}_{2}, \cdots, \widetilde{A}_{n}$ and $\widetilde{B}$ are fuzzy concepts. A fuzzy concept $\widetilde{B}$ is a logical consequent of $\widetilde{A}_{1}, \widetilde{A}_{2}, \cdots, \widetilde{A}_{n}$ if and only if the following inequality holds

$$
T\left(\bigcap_{\forall i \in[1, n]} \widetilde{A}_{i}\right)<T(\widetilde{B}) \text {. }
$$

Or equally

$$
\left\{\left[\widetilde{A}_{1} \text { and } \widetilde{A_{2}} \text { and } \cdots \text { and } \widetilde{A_{n}}\right] \Rightarrow \widetilde{B}\right\}
$$

Which means a fuzzy formula (2.30) is $U V$. Note, that a fuzzy formula $f \in F$ is called $U V$, if $T(f) \geq 0.5$. Before providing a proof of this theorem let us give the following

\section{Definition 2}

A fuzzy concept $\widetilde{B}$ is a logical consequent of $\widetilde{A}_{1}, \widetilde{A}_{2}, \cdots, \widetilde{A_{n}}$, i.e. $\widetilde{A_{1}}, \widetilde{A}_{2}, \cdots, \widetilde{A}_{n}=\widetilde{B}$, if and only when $U V$ of $\widetilde{A}_{1}, \widetilde{A}_{2}, \cdots, \widetilde{A}_{n}$ has caused $U V$ of a fuzzy concept $\widetilde{B}$, in other words the following is true.

$$
T\left(\bigcap_{\forall i \in[1, n]} \widetilde{A}_{i}\right) \geq 0.5 \Rightarrow T(\widetilde{B}) \geq 0.5, \text { where } \bigcap_{\forall i \in[1, n]} \widetilde{A}_{i}=\min _{i}\left\{\widetilde{A}_{i}\right\}
$$

Proof: Let fuzzy concept $\widetilde{B}$ is a logical consequent of fuzzy concepts $\widetilde{A_{1}}, \widetilde{A_{2}}, \cdots, \widetilde{A_{n}}$.

If fuzzy concepts $\widetilde{A_{1}}, \widetilde{A}_{2}, \cdots, \widetilde{A}_{n}$ are $U V$, i.e. $T\left(\widetilde{A}_{i}\right) \geq 0.5, i=1, n$, then in accordance with Definition 2 a fuzzy concept $\widetilde{B}$ is also $U V$, i.e. $T(\widetilde{B}) \geq 0.5$. An implication operator in a fuzzy logic, used in this article is defined as the following (see Table S1 and Table S2)

$$
T\left(\bigcap_{\forall i \in[1, n]} \widetilde{A}_{i}\right) \rightarrow T(\widetilde{B})=\left\{\begin{array}{l}
1, T\left(\bigcap_{\forall i \in[1, n]} \widetilde{A}_{i}\right) \leq T(\widetilde{B}), \\
\neg T\left(\bigcap_{\forall i \in[1, n]} \widetilde{A}_{i}\right) \cdot T(\widetilde{B}), T\left(\bigcap_{\forall i \in[1, n]} \widetilde{A}_{i}\right)>T(\widetilde{B})
\end{array}\right.
$$

Let us consider a set of cases.

- If $T\left(\bigcap_{\forall i \in[1, n]} \widetilde{A}_{i}\right) \leq T(\widetilde{B})$, then $T\left(\bigcap_{\forall i \in[1, n]} \widetilde{A}_{i}\right) \rightarrow T(\widetilde{B}) \equiv 1$, therefore $U V$ of a fuzzy formula (2.30) is apparent.

- If $T\left(\bigcap_{\forall i \in[1, n]} \widetilde{A}_{i}\right)>T(\widetilde{B})$, then $T\left(\bigcap_{\forall i \in[1, n]} \widetilde{A}_{i}\right) \rightarrow T(\widetilde{B}) \equiv \neg T\left(\bigcap_{\forall i \in[1, n]} \widetilde{A}_{i}\right) \cdot T(\widetilde{B})$, but since $T\left(\widetilde{A}_{i}\right) \geq 0.5, i=\overline{1, n}$ and $T(\widetilde{B}) \geq 0.5$, then $\neg T\left(\widetilde{A}_{i}\right)<0.5, i=\overline{1, n}$ and $\neg T\left(\bigcap_{\forall i \in[1, n]} \widetilde{A}_{i}\right)<T(\widetilde{B})$. Therefore it is obvious then 
$\neg T\left(\bigcap_{\forall i \in[1, n]} \widetilde{A}_{i}\right) \cdot T(\widetilde{B})<0.5$, i.e. a fuzzy formula $(2.30)$ is not $U V$, a logical contradiction takes place.

- If $\exists i=i^{*} \mid T\left(\widetilde{A_{i}^{*}}\right)<0.5$, then $T\left(\bigcap_{i} \widetilde{A_{i}}\right)=\min _{i=1, n}\left\{T\left(\widetilde{A_{i}}\right)\right\}<0.5$ a fuzzy sub formula (antecedent) from (2.30) $\left[\widetilde{A}_{1}\right.$ and $\widetilde{A}_{2}$ and $\cdots$ and $\left.\widetilde{A_{n}}\right]$ is not $U V$, i.e. contradictive, but in a meantime $T(\widetilde{B}) \geq 0.5$, therefore $T\left(\bigcap_{\forall i \in[1, n]} \widetilde{A}_{i}\right) \leq T(\widetilde{B}) \Rightarrow T\left(\bigcap_{\forall i \in[1, n]} \widetilde{A}_{i}\right) \rightarrow T(\widetilde{B}) \equiv 1$, i.e. a fuzzy formula $(2.30)$ is $U V$.

- If

$$
T\left(\bigcap_{\forall i \in[1, n]} \widetilde{A}_{i}\right)<0.5, T(\widetilde{B})<0.5
$$

and if $T\left(\bigcap_{\forall i \in[1, n]} \widetilde{A}_{i}\right) \leq T(\widetilde{B}) \Rightarrow T\left(\bigcap_{\forall i \in[1, n]} \widetilde{A}_{i}\right) \rightarrow T(\widetilde{B}) \equiv 1$, therefore formula (2.30) is $U V$, whereas if $T\left(\bigcap_{\forall i \in[1, n]} \widetilde{A}_{i}\right)>T(\widetilde{B})$, then again $T\left(\bigcap_{\forall i \in[1, n]} \widetilde{A}_{i}\right) \rightarrow T(\widetilde{B}) \equiv \neg T\left(\bigcap_{\forall i \in[1, n]} \widetilde{A}_{i}\right) \cdot T(\widetilde{B})$ and given conditions (2.32) we have $\neg T\left(\bigcap_{\forall i \in[1, n]} \widetilde{A}_{i}\right) \geq 0.5 \Rightarrow \neg T\left(\bigcap_{\forall i \in[1, n]} \widetilde{A}_{i}\right) \cdot T(\widetilde{B}) \leq 0.5$, which means that a fuzzy formula (2.30) is not $U V$ or is contradictive.

At last let a fuzzy formula (2.30) be $U V$ and also let $T\left(\bigcap_{\forall i \in[1, n]} \widetilde{A}_{i}\right) \leq T(\widetilde{B})$. Then if a fuzzy sub formula (antecedent) from (2.30) $\left[\widetilde{A}_{1}\right.$ and $\widetilde{A}_{2}$ and $\cdots$ and $\left.\widetilde{A}_{n}\right]$ is also $U V$, i.e. $T\left(\bigcap_{\forall i \in[1, n]} \widetilde{A}_{i}\right) \geq 0.5$, then from $(2.31)$ we have $T\left(\bigcap_{\forall i \in[1, n]} \widetilde{A}_{i}\right) \rightarrow T(\widetilde{B}) \equiv 1 \Rightarrow T(\widetilde{B}) \geq 0.5$. In other words a fuzzy concept $\widetilde{B}$ is $U V$. Therefore from Definition 2 a fuzzy concept $\widetilde{B}$ is a logical consequent of fuzzy concepts $\widetilde{A_{1}}, \widetilde{A_{2}}, \cdots, \widetilde{A_{n}}$ (Q. E. D.).

Based on these results we formulate the following

\section{Theorem 2}

If there are two fuzzy clauses $\widetilde{C_{1}}, \widetilde{C_{2}}$ and $R\left(\widetilde{C_{1}}, \widetilde{C_{2}}\right)$ is a fuzzy resolvent of them with keyword $x_{i}$, then $R\left(\widetilde{C_{1}}, \widetilde{C_{2}}\right)$ is logical consequent of both $\widetilde{C_{1}}$ and $\widetilde{C_{2}}$ i.e.

$$
\widetilde{C_{1}}, \widetilde{C_{2}} \mid=R\left(\widetilde{C_{1}}, \widetilde{C_{2}}\right)
$$




\section{Proof:}

Let $\widetilde{C_{1}}=x_{i} \vee L_{1}$ and $\widetilde{C_{2}}=\neg x_{i} \vee L_{2}$, where $L_{1}, L_{2} \neq 0$, whereas $R\left(\widetilde{C_{1}}, \widetilde{C_{2}}\right)=L_{1} \vee L_{2}$. By Definition 1 and in accordance with (2.5)

$$
\begin{gathered}
T\left(R\left(\widetilde{C_{1}}, \widetilde{C_{2}}\right)\right)=\left\{\begin{array}{l}
L_{1} \cdot L_{2}, L_{1}+L_{2}<1, \\
1, L_{1}+L_{2} \geq 1
\end{array}\right. \\
T\left(\widetilde{C_{1}}\right)=\left\{\begin{array}{l}
x_{i} \cdot L_{1}, x_{i}+L_{1}<1, \\
1, x_{i}+L_{1} \geq 1
\end{array}\right. \\
T\left(\widetilde{C_{2}}\right)=\left\{\begin{array}{l}
\neg x_{i} \cdot L_{2}, \neg x_{i}+L_{2}<1, \\
1, \neg x_{i}+L_{2} \geq 1
\end{array}\right.
\end{gathered}
$$

Let $\widetilde{C_{1}}, \widetilde{C_{2}}$ are both $U V$, i.e. $T\left(\widetilde{C_{1}}\right) \geq 0.5$ and $T\left(\widetilde{C_{2}}\right) \geq 0.5$. Since from (2.35) and (2.36) the following is taking place $x_{i} \cdot L_{1}<0.5 \mid x_{i}+L_{1}<1$ and $\neg x_{i} \cdot L_{2}<0.5 \mid \neg x_{i}+L_{2}<1$ then $U V$ of $\widetilde{C_{1}}, \widetilde{C_{2}}$ is in reality means that

$$
\left.\begin{array}{l}
x_{i}+L_{1} \geq 1 \\
\neg x_{i}+L_{2} \geq 1
\end{array}\right\}
$$

Taking into account that $T\left(\neg x_{i}\right)=1-T\left(x_{i}\right)$ let sum both inequalities (2.37) together and get the following $L_{1}+L_{2} \geq 1$. From (2.34)

$T\left(R\left(\widetilde{C_{1}}, \widetilde{C_{2}}\right)\right)=1 \mid L_{1}+L_{2} \geq 1 \Rightarrow T\left(R\left(\widetilde{C_{1}}, \widetilde{C_{2}}\right)\right) \geq 0.5 \quad$ i.e. $\quad R\left(\widetilde{C_{1}}, \widetilde{C_{2}}\right) \quad$ is $\quad U V$. Therefore by Definition 2 we are getting a fact that if $\widetilde{C_{1}}, \widetilde{C}_{2}$ are both $U V$, and then $R\left(\widetilde{C}_{1}, \widetilde{C_{2}}\right)$ is also $U V$. (Q. E. D.).

Let us present some considerations about using a notion of similarity, which plays a fundamental role in theories of knowledge and behavior and has been dealt with extensively in psychology and philosophy. A careful analysis of the different similarity measures reveals that it is impossible to single out one particular similarity measure that works well for all purposes. We will utilize a consistent approach toward definition of a similarity measure, based on the same fuzzy logic we used above [1]. But this time we will use the operation Equivalence (see Table S1).

Suppose $U$ be an arbitrary finite set, and $\mathfrak{I}(U)$ be the collection of all fuzzy subsets of $U$. For $A, B \in \mathfrak{I}(U)$, a similarity index between the pair $\{A, B\}$ is denoted as $S(A, B ; U)$ or simply $S(A, B)$ which can also be considered as a function $S: \mathfrak{I}(U) \times \mathfrak{I}(U) \rightarrow[0,1]$. In order to provide a definition for similarity index, a number of factors must be considered.

\section{Definition 3}

A function $S(A, B)$ defines a similarity between fuzzy concepts $A, B$ if it satisfies the following axioms:

P1. $S(B, A)=S(A, B), S(\neg A, \neg B)=S(A, B)$, where $\neg A=1-A$,

P2. $0 \leq S(A, B) \leq 1$,

P3. $S(A, B)=1$,iff $A=B$,

P4. For two fuzzy concepts $A, B \mid A \neq \varnothing, B \neq \varnothing$, $S(A, B)=0 \Rightarrow \min \left(\mu_{A}(u), \mu_{B}(u)\right)=0, \forall u \in U$, i.e. $A \cap B=\varnothing$,

P5. $A \subseteq B \subseteq C(A \supseteq B \supseteq C) \Rightarrow S(A, C) \leq \min (S(A, B), S(B, C))$. 


\section{Lemma 2}

If a function $S(A, B)$ is defined as operation equivalence from Table $\mathrm{S} 1$, then it could be considered as a similarity measure.

\section{Proof:}

From Table S1 we have

$$
S(A, B)=\left\{\begin{array}{l}
(1-b) \cdot a, a<b \\
1, a=b \\
(1-a) \cdot b, a>b
\end{array}\right.
$$

P1. From (2.38)

$$
S(\neg A, \neg B)=\left\{\begin{array}{l}
b \cdot \neg a, \neg a<b, \\
1, \neg a=\neg b, \\
a \cdot \neg b, \neg a>b,
\end{array}=\left\{\begin{array}{l}
(1-a) \cdot b, a>b, \\
1, a=b, \\
(1-b) \cdot a, a<b,
\end{array} \Rightarrow S(\neg A, \neg B)=S(A, B)\right.\right.
$$

whereas

$$
S(B, A)=\left\{\begin{array}{l}
b \cdot(1-a), b<a, \\
1, b=a, \\
(1-b) \cdot a, b>a,
\end{array}=S(A, B)\right.
$$

Axioms P2 and P3 are trivially satisfied by (2.38).

P4. From (2.38)

$$
S(A, B) \equiv 0 \mid b=1, a=0 \text { or } b=0, a=1 \Rightarrow A \cap B=\varnothing
$$

P5. From (2.38)

$$
S(B, C)=\left\{\begin{array}{l}
(1-c) \cdot b, b<c \\
1, b=c \\
(1-b) \cdot c, b>c
\end{array}\right.
$$

Case: $a<b<c$

From (2.38) and (2.42) we have

$$
S(A, B)=(1-b) \cdot a ; S(B, C)=(1-c) \cdot b ; S(A, C)=(1-c) \cdot a
$$

From $(2.43)(1-c) \cdot a<(1-c) \cdot b \Rightarrow S(A, C)<S(B, C)$, whereas $(1-c) \cdot a<(1-b) \cdot a \Rightarrow S(A, C)<S(A, B)$. Since $S(A, C)<S(B, C)$ and $S(A, C)<S(A, B)$, then the following is also true:

$$
S(A, C) \leq \min (S(A, B), S(B, C))
$$

Case: $a>b>c$

From (2.38) and (2.42) we have

$$
S(A, B)=(1-a) \cdot b ; S(B, C)=(1-b) \cdot c ; S(A, C)=(1-a) \cdot c
$$

From $(2.45)(1-a) \cdot c<(1-a) \cdot b \Rightarrow S(A, C)<S(A, B)$, whereas $(1-a) \cdot c<(1-b) \cdot c \Rightarrow S(A, C)<S(A, B)$. Since $S(A, C)<S(B, C)$ and $S(A, C)<S(A, B)$, then the following is also true:

$$
S(A, C) \leq \min (S(A, B), S(B, C)) \quad \text { (Q. E. D.). }
$$


To illustrate our further research before giving the definition of similarity index, we will present couple examples.

Let $A$ and $B$ be two normal fuzzy sets defined over the same universe of discourse $U$ and presented by unimodal linear monotonic membership functions and $\operatorname{supp}\{A\} \geq \operatorname{Card} U-1 ; \operatorname{supp}\{B\} \geq \operatorname{Card} U-1$. Correspondent linguistic scale could consist of the terms like \{ $S M A L L " . .$, " $M E D I U M$ "..., " $L A R G E$ " . Let us consider the following cases.

1) $A$ labeled "SMALLER THAN LARGE"

$=0.2 / u_{1}+0.3 / u_{2}+0.4 / u_{3}+0.5 / u_{4}+0.6 / u_{5}+0.7 / u_{6}$

$+0.8 / u_{7}+0.9 / u_{8}+1.0 / u_{9}+0.9 / u_{10}+0.8 / u_{11}$

Whereas $B$ labeled "LARGE"

$=0.0 / u_{1}+0.1 / u_{2}+0.2 / u_{3}+0.3 / u_{4}+0.4 / u_{5}+0.5 / u_{6}$

$+0.6 / u_{7}+0.7 / u_{8}+0.8 / u_{9}+0.9 / u_{10}+1.0 / u_{11}$

From (2.38) the similarity matrix $S(A, B)$ would look like that

2) $\begin{aligned} A \text { labeled "MEDIUM" }= & 0.5 / u_{1}+0.6 / u_{2}+0.7 / u_{3}+0.8 / u_{4}+0.9 / u_{5}+1.0 / u_{6} \\ & +0.9 / u_{7}+0.8 / u_{8}+0.7 / u_{9}+0.6 / u_{10}+0.5 / u_{11}\end{aligned}$

And $B$ labeled "LARGE"

$=0.0 / u_{1}+0.1 / u_{2}+0.2 / u_{3}+0.3 / u_{4}+0.4 / u_{5}+0.5 / u_{6}$

$+0.6 / u_{7}+0.7 / u_{8}+0.8 / u_{9}+0.9 / u_{10}+1.0 / u_{11}$

From (2.38) the similarity matrix $S(A, B)$ would look like that (for simplicity sake we show elements of a matrix with singles only)

3) $\begin{aligned} A \text { labeled "MEDIUM" } \begin{aligned}= & 0.5 / u_{1}+0.6 / u_{2}+0.7 / u_{3}+0.8 / u_{4}+0.9 / u_{5}+1.0 / u_{6} \\ & +0.9 / u_{7}+0.8 / u_{8}+0.7 / u_{9}+0.6 / u_{10}+0.5 / u_{11}\end{aligned}\end{aligned}$

And $B$ labeled "SMALL"

$=1.0 / u_{1}+0.9 / u_{2}+0.8 / u_{3}+0.7 / u_{4}+0.6 / u_{5}+0.5 / u_{6}$

$+0.4 / u_{7}+0.3 / u_{8}+0.2 / u_{9}+0.1 / u_{10}+0.0 / u_{11}$

From (2.38) the similarity matrix $S(A, B)$ would look like that

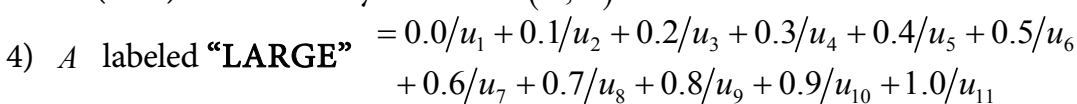

And $B$ labeled "SMALL"

$=1.0 / u_{1}+0.9 / u_{2}+0.8 / u_{3}+0.7 / u_{4}+0.6 / u_{5}+0.5 / u_{6}$

$+0.4 / u_{7}+0.3 / u_{8}+0.2 / u_{9}+0.1 / u_{10}+0.0 / u_{11}$

From (2.38) the similarity matrix $S(A, B)$ would look like that

5) $\begin{aligned} A \text { labeled “MEDIUM" }= & 0.5 / u_{1}+0.6 / u_{2}+0.7 / u_{3}+0.8 / u_{4}+0.9 / u_{5}+1.0 / u_{6} \\ & +0.9 / u_{7}+0.8 / u_{8}+0.7 / u_{9}+0.6 / u_{10}+0.5 / u_{11}\end{aligned}$

And $B$ labeled "MEDIUM"

$=0.5 / u_{1}+0.6 / u_{2}+0.7 / u_{3}+0.8 / u_{4}+0.9 / u_{5}+1.0 / u_{6}$

$+0.9 / u_{7}+0.8 / u_{8}+0.7 / u_{9}+0.6 / u_{10}+0.5 / u_{11}$

From (2.38) the similarity matrix $S(A, B)$ would look like that

Let consider similarity measure as a matrix

$S(A, B)=\left\|s_{i j}\right\| ; i=\overline{1, n} ; j=\overline{1, n} ; n=\operatorname{Card} U$. We are presenting the following

Proposition 1

Since $A$ and $B$ are two normal fuzzy sets, then

$\exists i^{*}, j^{*} \in[1, n] \mid s_{i^{*} j^{*}}=1 ; n=\operatorname{Card} U$. Then the following function could be consi- 
dered as a similarity index

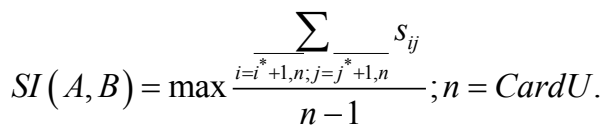

In Table 1 there are three sets of pairs of indices

$i, j \mid i \in\left[i^{*}+1, n\right] ; j \in\left[j^{*}+1, n\right] ; s_{i^{*} j^{*}}=1 ; n=$ CardU .

1) For $i^{*}=1, j^{*}=3, G r_{1}\{i, j\}=\{2,4 ; 3,5 ; 4,6 ; 5,7 ; 6,8 ; 7,9 ; 8,10 ; 9,11\}$

$\Rightarrow \operatorname{SI}\left(G r_{1}\{i, j\}\right)=\frac{8}{10}=0.8$

2) For $i^{*}=10, j^{*}=10, G r_{2}\{i, j\}=\varnothing \Rightarrow S I\left(G r_{2}\{i, j\}\right)=0$

3) For $i^{*}=11, j^{*}=9, G r_{3}\{i, j\}=\varnothing \Rightarrow \operatorname{SI}\left(G r_{3}\{i, j\}\right)=0$.

From (2.46) we are getting $S I(A, B)=\max _{k=1,3}\left[S I\left(G r_{k}\left\{i^{*}, j^{*}\right\}\right)\right]=0.8$. This value perfectly matches our intuition and perception of a closeness of terms "SMALLER THAN LARGE" and "LARGE" and membership functions of correspondent fuzzy sets.

In Table 2 there are six sets of pairs of indices

$i, j \mid i \in\left[i^{*}+1, n\right] ; j \in\left[j^{*}+1, n\right] ; s_{i^{*} j^{*}}=1 ; n=$ Card $U$.

1) For $i^{*}=1, j^{*}=6, G r_{1}\{i, j\}=\{2,7 ; 3,8 ; 4,9 ; 5,10 ; 6,11\}$

$\Rightarrow \operatorname{SI}\left(G r_{1}\{i, j\}\right)=\frac{5}{10}=0.5$

2) For $i^{*}=7, j^{*}=10, G r_{2}\{i, j\}=\varnothing \Rightarrow S I\left(G r_{2}\{i, j\}\right)=0$

3) For $i^{*}=8, j^{*}=9, G r_{3}\{i, j\}=\varnothing \Rightarrow \operatorname{SI}\left(G r_{3}\{i, j\}\right)=0$

4) For $i^{*}=9, j^{*}=8, G r_{4}\{i, j\}=\varnothing \Rightarrow \operatorname{SI}\left(G r_{4}\{i, j\}\right)=0$

5) For $i^{*}=10, j^{*}=7, G r_{5}\{i, j\}=\varnothing \Rightarrow \operatorname{SI}\left(G r_{5}\{i, j\}\right)=0$

6) For $i^{*}=11, j^{*}=6, G r_{6}\{i, j\}=\varnothing \Rightarrow \operatorname{SI}\left(G r_{6}\{i, j\}\right)=0$

Table 1. "Smaller Than Large" $\Rightarrow$ "Large".

\begin{tabular}{cccccccccccc}
\hline$S(A, B)$ & $\mathbf{0}$ & $\mathbf{0 . 1}$ & $\mathbf{0 . 2}$ & $\mathbf{0 . 3}$ & $\mathbf{0 . 4}$ & $\mathbf{0 . 5}$ & $\mathbf{0 . 6}$ & $\mathbf{0 . 7}$ & $\mathbf{0 . 8}$ & $\mathbf{0 . 9}$ & $\mathbf{1}$ \\
\hline $\mathbf{0 . 2}$ & 0 & 0.08 & $\mathbf{1}$ & 0.14 & 0.12 & 0.1 & 0.08 & 0.06 & 0.04 & 0.02 & 0 \\
$\mathbf{0 . 3}$ & 0 & 0.07 & 0.14 & $\mathbf{1}$ & 0.18 & 0.15 & 0.12 & 0.09 & 0.06 & 0.03 & 0 \\
$\mathbf{0 . 4}$ & 0 & 0.06 & 0.12 & 0.18 & $\mathbf{1}$ & 0.2 & 0.16 & 0.12 & 0.08 & 0.04 & 0 \\
$\mathbf{0 . 5}$ & 0 & 0.05 & 0.1 & 0.15 & 0.2 & $\mathbf{1}$ & 0.2 & 0.15 & 0.1 & 0.05 & 0 \\
$\mathbf{0 . 6}$ & 0 & 0.04 & 0.08 & 0.12 & 0.16 & 0.2 & $\mathbf{1}$ & 0.18 & 0.12 & 0.06 & 0 \\
$\mathbf{0 . 7}$ & 0 & 0.03 & 0.06 & 0.09 & 0.12 & 0.15 & 0.18 & $\mathbf{1}$ & 0.14 & 0.07 & 0 \\
$\mathbf{0 . 8}$ & 0 & 0.02 & 0.04 & 0.06 & 0.08 & 0.1 & 0.12 & 0.14 & $\mathbf{1}$ & 0.08 & 0 \\
$\mathbf{0 . 9}$ & 0 & 0.01 & 0.02 & 0.03 & 0.04 & 0.05 & 0.06 & 0.07 & 0.08 & $\mathbf{1}$ & 0 \\
$\mathbf{1}$ & 0 & 0 & 0 & 0.0 & 0 & 0 & 0 & 0.0 & 0 & 0 & $\mathbf{1}$ \\
$\mathbf{0 . 9}$ & 0 & 0.01 & 0.02 & 0.03 & 0.04 & 0.05 & 0.06 & 0.07 & 0.08 & $\mathbf{1}$ & 0 \\
$\mathbf{0 . 8}$ & 0 & 0.02 & 0.04 & 0.06 & 0.08 & 0.1 & 0.12 & 0.14 & $\mathbf{1}$ & 0.18 & 0 \\
\hline
\end{tabular}


Table 2. "Medium" $\Rightarrow$ "Large".

\begin{tabular}{|c|c|c|c|c|c|c|c|c|c|c|c|}
\hline$S(A, B)$ & 0 & 0.1 & 0.2 & 0.3 & 0.4 & 0.5 & 0.6 & 0.7 & 0.8 & 0.9 & 1 \\
\hline 0.5 & $\ldots$ & & & $\ldots$ & & 1 & & $\ldots$ & $\ldots$ & . & . \\
\hline 0.6 & & & & & & & 1 & . & . & . & . \\
\hline 0.7 & & $\ldots$ & & & & . & & 1 & . & . & . \\
\hline 0.8 & & & & & & & & & 1 & . & . \\
\hline 0.9 & & & & & & & & & & 1 & . \\
\hline 1 & & & & & & & . & & & & 1 \\
\hline 0.9 & & & & & & & & & & 1 & \\
\hline 0.8 & & & & & & & & & 1. & & \\
\hline 0.7 & & & & & & & & 1 & & & \\
\hline 0.6 & & & & & & & 1 & & . & & \\
\hline 0.5 & & & & & & 1 & & & & & \\
\hline
\end{tabular}

Table 3. "Medium" $\Rightarrow$ "Small".

\begin{tabular}{|c|c|c|c|c|c|c|c|c|c|c|c|}
\hline$S(A, B)$ & 1 & 0.9 & 0.8 & 0.7 & 0.6 & 0.5 & 0.4 & 0.3 & 0.2 & 0.1 & 0 \\
\hline 0.5 & $\ldots$ & & & $\ldots$ & & 1 & & $\ldots$ & $\ldots$ & . & . \\
\hline 0.6 & & & & & 1 & & & . & . & . & . \\
\hline 0.7 & & $\ldots$ & & 1 & & . & & & . & . & . \\
\hline 0.8 & & & 1 & & & & & & & . & . \\
\hline 0.9 & & 1 & & & & & & & & & . \\
\hline 1 & 1 & & & & & & . & & & & \\
\hline 0.9 & & 1 & & & & & & & & & \\
\hline 0.8 & & & 1 & & & & & & . & & \\
\hline 0.7 & & & & 1 & & & & & & & \\
\hline 0.6 & & & & & 1 & & & & . & & \\
\hline 0.5 & & & & & & 1 & & & & & \\
\hline
\end{tabular}

From (2.46) $S I(A, B)=\max _{k=1,6}\left[S I\left(G r_{k}\left\{i^{*}, j^{*}\right\}\right)\right]=0.5$. This value is in a middle of a scale $[0,1]$ and also perfectly matches our intuition and perception of an average closeness of terms " $L A R G E$ " and "MEDIUM" and membership functions of correspondent fuzzy sets.

Similarly in Table 3 there are six sets of pairs of indices

$i, j \mid i \in\left[i^{*}+1, n\right] ; j \in\left[j^{*}+1, n\right] ; s_{i^{*} j^{*}}=1 ; n=C$ Card $U$.

1) For $i^{*}=6, j^{*}=1, G r_{1}\{i, j\}=\{7,2 ; 8,3 ; 9,4 ; 10,5 ; 11,6\}$

$\Rightarrow S I\left(G r_{1}\{i, j\}\right)=\frac{5}{10}=0.5$

2) For $i^{*}=5, j^{*}=2, G r_{2}\{i, j\}=\varnothing \Rightarrow S I\left(G r_{2}\{i, j\}\right)=0$

3) For $i^{*}=4, j^{*}=3, G r_{3}\{i, j\}=\varnothing \Rightarrow S I\left(G r_{3}\{i, j\}\right)=0$ 
4) For $i^{*}=3, j^{*}=4, G r_{4}\{i, j\}=\varnothing \Rightarrow \operatorname{SI}\left(G r_{4}\{i, j\}\right)=0$

5) For $i^{*}=2, j^{*}=5, G r_{5}\{i, j\}=\varnothing \Rightarrow S I\left(G r_{5}\{i, j\}\right)=0$

6) For $i^{*}=1, j^{*}=6, G r_{6}\{i, j\}=\varnothing \Rightarrow \operatorname{SI}\left(G r_{6}\{i, j\}\right)=0$.

From (2.46) we are getting $S I(A, B)=\max _{k=1,6}\left[S I\left(G r_{k}\left\{i^{*}, j^{*}\right\}\right)\right]=0.5$. This value is also in a middle of a scale $[0,1]$ and also perfectly matches our intuition and perception of an average closeness of terms "SMALL" and "MEDIUM" and membership functions of correspondent fuzzy sets.

In Table 4 there are eleven sets of pairs of indices $i, j \mid i \in\left[i^{*}+1, n\right] ; j \in\left[j^{*}+1, n\right] ; s_{i^{*} j^{*}}=1 ; n=\operatorname{Card} U$.

1) For $i^{*}=11, j^{*}=1, G r_{1}\{i, j\}=\varnothing \Rightarrow S I\left(G r_{1}\{i, j\}\right)=0$

2) $i^{*}=10, j^{*}=2, G r_{2}\{i, j\}=\varnothing \Rightarrow S I\left(G r_{2}\{i, j\}\right)=0$

3) $i^{*}=9, j^{*}=3, G r_{3}\{i, j\}=\varnothing \Rightarrow \operatorname{SI}\left(G r_{3}\{i, j\}\right)=0$

4) $i^{*}=8, j^{*}=4, G r_{4}\{i, j\}=\varnothing \Rightarrow \operatorname{SI}\left(G r_{4}\{i, j\}\right)=0$

5) $i^{*}=7, j^{*}=5, G r_{5}\{i, j\}=\varnothing \Rightarrow \operatorname{SI}\left(G r_{5}\{i, j\}\right)=0$

6) $i^{*}=6, j^{*}=6, G r_{6}\{i, j\}=\varnothing \Rightarrow S I\left(G r_{6}\{i, j\}\right)=0$

7) For $i^{*}=5, j^{*}=7, G r_{7}\{i, j\}=\varnothing \Rightarrow \operatorname{SI}\left(G r_{7}\{i, j\}\right)=0$

8) For $i^{*}=4, j^{*}=8, G r_{8}\{i, j\}=\varnothing \Rightarrow S I\left(G r_{8}\{i, j\}\right)=0$

9) For $i^{*}=3, j^{*}=9, G r_{9}\{i, j\}=\varnothing \Rightarrow S I\left(G r_{9}\{i, j\}\right)=0$

10) For $i^{*}=2, j^{*}=10, G r_{10}\{i, j\}=\varnothing \Rightarrow \operatorname{SI}\left(G r_{10}\{i, j\}\right)=0$

11) For $i^{*}=1, j^{*}=11, G r_{11}\{i, j\}=\varnothing \Rightarrow S I\left(G r_{11}\{i, j\}\right)=0$.

From (2.46) we are getting $\operatorname{SI}(A, B)=\max _{k=11}\left[S I\left(G r_{k}\left\{i^{*}, j^{*}\right\}\right)\right]=0$. This value also perfectly matches our intuition and perception of a fact that terms "SMALL" and "LARGE" has nothing in common.

Table 4. "Large" $\Rightarrow$ "Small".

\begin{tabular}{|c|c|c|c|c|c|c|c|c|c|c|c|}
\hline$S(A, B)$ & 1 & 0.9 & 0.8 & 0.7 & 0.6 & 0.5 & 0.4 & 0.3 & 0.2 & 0.1 & 0 \\
\hline 0 & $\ldots$ & & & $\ldots$ & & & & $\ldots$ & $\ldots$ & . & 1. \\
\hline 0.1 & & & & & & & & . & . & 1. & . \\
\hline 0.2 & & $\ldots$ & & & & . & & & 1. & . & . \\
\hline 0.3 & & & & & & & & 1 & & . & . \\
\hline 0.4 & & & & & & & 1 & & & & . \\
\hline 0.5 & & & & & & 1 & & & & & \\
\hline 0.6 & & & & & 1 & & & & & & \\
\hline 0.7 & & & & 1 & & & & & . & & \\
\hline 0.8 & & & 1 & & & & & & & & \\
\hline 0.9 & & 1 & & & & & & & . & & \\
\hline 1 & 1 & & & & & & & & & & \\
\hline
\end{tabular}


Table 5. "Medium" $\Rightarrow$ "Medium".

\begin{tabular}{|c|c|c|c|c|c|c|c|c|c|c|c|}
\hline$S(A, B)$ & 0.5 & 0.6 & 0.7 & 0.8 & 0.9 & 1 & 0.9 & 0.8 & 0.7 & 0.6 & 0.5 \\
\hline 0.5 & 1 & & & $\ldots$ & & & & $\ldots$ & $\ldots$ & . & 1. \\
\hline 0.6 & & 1 & & & & & & . & . & 1. & . \\
\hline 0.7 & & $\ldots$ & 1 & & & . & & & 1. & . & . \\
\hline 0.8 & & & & 1 & & & & 1 & & . & . \\
\hline 0.9 & & & & & 1 & & 1 & & & & . \\
\hline 1 & & & & & & 1 & . & & & & \\
\hline 0.9 & & & & & 1 & & 1 & & & & \\
\hline 0.8 & & & & 1 & & & & 1 & . & & \\
\hline 0.7 & & & 1 & & & & & & 1 & & \\
\hline 0.6 & & 1 & & & & & & & . & 1 & \\
\hline 0.5 & 1 & & & & & & & & & & 1 \\
\hline
\end{tabular}

In Table 5 there are twelve sets of pairs of indices

$i, j \mid i \in\left[i^{*}+1, n\right] ; j \in\left[j^{*}+1, n\right] ; s_{i^{*} j^{*}}=1 ; n=$ Card $U$.

1) For $i^{*}=11, j^{*}=1, G r_{1}\{i, j\}=\varnothing \Rightarrow \operatorname{SI}\left(G r_{1}\{i, j\}\right)=0$

2) $i^{*}=10, j^{*}=2, G r_{2}\{i, j\}=\varnothing \Rightarrow S I\left(G r_{2}\{i, j\}\right)=0$

3) $i^{*}=9, j^{*}=3, G r_{3}\{i, j\}=\varnothing \Rightarrow \operatorname{SI}\left(G r_{3}\{i, j\}\right)=0$

4) $i^{*}=8, j^{*}=4, G r_{4}\{i, j\}=\varnothing \Rightarrow S I\left(G r_{4}\{i, j\}\right)=0$

5) $i^{*}=7, j^{*}=5, G r_{5}\{i, j\}=\varnothing \Rightarrow S I\left(G r_{5}\{i, j\}\right)=0$

6) $i^{*}=6, j^{*}=6, G r_{6}\{i, j\}=\varnothing \Rightarrow \operatorname{SI}\left(G r_{6}\{i, j\}\right)=0$

7) For $i^{*}=5, j^{*}=7, G r_{7}\{i, j\}=\varnothing \Rightarrow \operatorname{SI}\left(G r_{7}\{i, j\}\right)=0$

8) For $i^{*}=4, j^{*}=8, G r_{8}\{i, j\}=\varnothing \Rightarrow \operatorname{SI}\left(G r_{8}\{i, j\}\right)=0$

9) For $i^{*}=3, j^{*}=9, G r_{9}\{i, j\}=\varnothing \Rightarrow \operatorname{SI}\left(G r_{9}\{i, j\}\right)=0$

10) For $i^{*}=2, j^{*}=10, G r_{10}\{i, j\}=\varnothing \Rightarrow S I\left(G r_{10}\{i, j\}\right)=0$

11) For $i^{*}=1, j^{*}=11, G r_{11}\{i, j\}=\varnothing \Rightarrow \operatorname{SI}\left(G r_{11}\{i, j\}\right)=0$.

12) For $i^{*}=1, j^{*}=1, G r_{12}\{i, j\}=\{2,2 ; 3,3 ; 4,4 ; 5,5 ; 6,6 ; 7,7 ; 8,8 ; 9,9 ; 10,10 ; 11,11\}$ $\Rightarrow \operatorname{SI}\left(G r_{12}\{i, j\}\right)=\frac{10}{10}=1$.

From (2.46) we are getting $\operatorname{SI}(A, B)=\max _{k=12}\left[S I\left(G r_{k}\left\{i^{*}, j^{*}\right\}\right)\right]=1$. This value is a confirmation of a fact that both fuzzy sets are identical.

\section{Generalized Fuzzy Resolution Based Approximate Reasoning}

Let us remind that the scheme for Generalized Fuzzy Resolution (2.3) looks like that 


\section{Antecedent1: If $x$ is $A O R y$ is $B$ \\ Antecedent2: $y$ is $B$ ' \\ Consequent: $x$ is $A$ '.}

First consider the following classical logic equivalence

$$
a \vee b \equiv b \vee a \equiv \neg b \rightarrow a
$$

The classical logic equivalence (3.2) can be extended in fuzzy logic with implication and negation functions. We use the same fuzzy logic, which operations are presented in Table S1. Let us first proof that (3.2) holds.

Since

$$
\begin{gathered}
\qquad a \vee b=\left\{\begin{array}{l}
a \cdot b, a+b<1, \\
1, a+b \geq 1
\end{array} \Rightarrow b \vee a=\left\{\begin{array}{l}
b \cdot a, b+a<1, \\
1, b+a \geq 1
\end{array} \Rightarrow a \vee b \equiv b \vee a\right.\right. \\
\text { And because } a \rightarrow b=\left\{\begin{array}{l}
(1-a) \cdot b, a>b, \\
1, a \leq b
\end{array}\right. \text { therefore } \\
\neg b \rightarrow a=\left\{\begin{array}{l}
(1-\neg b) \cdot a, \neg b>a, \\
1, \neg b \leq a
\end{array}=\left\{\begin{array}{l}
b \cdot a, b+a<1, \\
1, b+a \geq 1
\end{array} \equiv a \vee b\right.\right.
\end{gathered}
$$

Both (3.3) and (3.4) proofs that classical logic equivalence (3.2) holds. It is very important to show that we transform Generalized Fuzzy Resolution rule (3.1) into its equivalent form

$$
\begin{gathered}
\text { Antecedent } 1 \text { If } y \text { is } \neg B \text { then } x \text { is } A \\
\text { Antecedent2: } y \text { is } B^{\prime} \\
\text { Consequent: } x \text { is } A^{\prime} \text {. }
\end{gathered}
$$

Let us formulize an inference method for a rule (3.5). Following a well-known pattern, established a couple of decades ago and the standard approaches toward such formalization, presented and extensively used in [5] [6] [7] [8] [9], let $U$ and $V$ be two universes of discourses and correspondent fuzzy sets be represented as such $A \subset U\left|\mu_{A}: U \rightarrow[0,1], B \subset V\right| \mu_{B}: V \rightarrow[0,1]$, where

$$
A=\int_{U} \mu_{A}(u) / u, B=\int_{V} \mu_{B}(v) / v
$$

Whereas given (3.6) a binary relationship for the fuzzy conditional proposition of the type: "If $y$ is $\neg B$ then $x$ is $A$ " for a fuzzy logic is defined as

$$
\begin{aligned}
& R\left(D_{1}(y), D_{2}(x)\right) \\
= & \neg B \times V \rightarrow U \times A \\
= & \int_{V \times U}\left(1-\mu_{B}(v)\right) /(v, u) \rightarrow \int_{V \times U} \mu_{A}(u) /(v, u) \\
= & \int_{V \times U}\left(\left(1-\mu_{B}(v)\right) \rightarrow \mu_{A}(u)\right) /(v, u)
\end{aligned}
$$

Given an implication operator from Table S1 expression (3.7) looks like 


$$
\begin{aligned}
& \left(1-\mu_{B}(v)\right) \rightarrow \mu_{A}(u)=\left\{\begin{array}{l}
\mu_{B}(v) \cdot \mu_{A}(u), 1-\mu_{B}(v)>\mu_{A}(u), \\
1,1-\mu_{B}(v) \leq \mu_{A}(u) .
\end{array}\right. \\
& =\left\{\begin{array}{l}
\mu_{B}(v) \cdot \mu_{A}(u), \mu_{A}(u)+\mu_{B}(v)<1, \\
1, \mu_{A}(u)+\mu_{B}(v) \geq 1 .
\end{array}\right.
\end{aligned}
$$

It is well known that given a unary relationship $R\left(D_{1}(y)\right)=B^{\prime}$ one can obtain the consequence $R\left(D_{2}(x)\right)$ by applying compositional rule of inference (CRI) to $R\left(D_{1}(y)\right)$ and $R\left(D_{1}(y), D_{2}(x)\right)$ of type (3.7):

$$
\begin{aligned}
R\left(D_{2}(x)\right) & =R\left(D_{1}(y)\right) \circ R\left(D_{1}(y), D_{2}(x)\right) \\
& =\int_{V} \mu_{B^{\prime}}(v) / v \circ \int_{V \times U}\left(\left(1-\mu_{B}(v)\right) \rightarrow \mu_{A}(u)\right) /(v, u) \\
& =\int_{U} \bigcup_{v \in V}\left[\mu_{B^{\prime}}(v) \wedge\left(\left(1-\mu_{B}(v)\right) \rightarrow \mu_{A}(u)\right)\right] / v
\end{aligned}
$$

In order that Criterion $I$ (see Appendix) is satisfied, that is $R\left(D_{2}(x)\right)=\neg A$ from (3.9) the equality

$$
\bigcup_{v \in V}\left[\mu_{B}(v) \wedge\left(\left(1-\mu_{B}(v)\right) \rightarrow \mu_{A}(u)\right)\right]=1-\mu_{A}(u)
$$

must be satisfied for arbitrary $u \in U$ and in order that the equality (3.10) is satisfied, it is necessary that the inequality

$$
\mu_{B}(v) \wedge\left(\left(1-\mu_{B}(v)\right) \rightarrow \mu_{A}(u)\right) \leq 1-\mu_{A}(u)
$$

holds for arbitrary $u \in U$ and $v \in V$. Let us define new methods of fuzzy conditional inference of the type (3.5), which requires the satisfaction of Criteria $I-I V$ from Appendix.

\section{Theorem 3}

If fuzzy sets $A \subset U\left|\mu_{A}: U \rightarrow[0,1], B \subset V\right| \mu_{B}: V \rightarrow[0,1]$ are defined as (3.6) and $R\left(D_{1}(y), D_{2}(x)\right)$ is defined by (3.7), where

$$
\left(1-\mu_{B}(v)\right) \rightarrow \mu_{A}(u)=\left\{\begin{array}{l}
\mu_{B}(v) \cdot \mu_{A}(u), \mu_{B}(v)<1-\mu_{A}(u), \\
1, \mu_{B}(v) \geq 1-\mu_{A}(u) .
\end{array}\right.
$$

then Criteria I, II, III and IV-1 are satisfied.

Proof:

For Criteria I-III let $R\left(D_{1}(y)\right)=B^{\alpha}(\alpha>0)$ then

$$
\begin{aligned}
& R\left(D_{2}(x)\right)=B^{\alpha} \circ R\left(D_{1}(y), D_{2}(x)\right) \\
& =\int_{V} \mu_{B}^{\alpha}(v) / v \circ \int_{V \times U}\left(\left(1-\mu_{B}(v)\right) \rightarrow \mu_{A}(u)\right) /(v, u) \\
& =\int_{U} \bigcup_{v \in V}\left[\mu_{B}^{\alpha}(v) \wedge\left(\left(1-\mu_{B}(v)\right) \rightarrow \mu_{A}(u)\right)\right] / u \\
& \exists V_{1}, V_{2} \subset V\left|V_{1} \cup V_{2}=V ;\right| V_{1} \cap V_{2}=\varnothing \\
& \Rightarrow \forall v \in V_{1}\left|\mu_{B}(v)<1-\mu_{A}(u) ; \forall v \in V_{2}\right| \mu_{B}(v) \geq 1-\mu_{A}(u)
\end{aligned}
$$

From (3.13) and given subsets from (3.14) we have

$$
R\left(D_{2}(x)\right)=\left[\int_{U} \bigcup_{v \in V_{1}}\left[\mu_{B}^{\alpha}(v) \wedge\left(\mu_{B}(v) \cdot \mu_{A}(u)\right)\right] / u\right] \vee\left[\int_{U} \bigcup_{v \in V_{2}}\left[\mu_{B}^{\alpha}(v) \wedge 1\right] / u\right] .
$$


Let us introduce the following function (as a part of implication operation)

$$
f(v, u)=\mu_{B}(v) \cdot \mu_{A}(u) \mid \mu_{B}(v)<1-\mu_{A}(u) .
$$

Then the following is taking place:

$$
\forall v \in V_{1} \mid \mu_{B}^{\alpha}(v) \wedge f(v, u)=\left\{\begin{array}{l}
\mu_{B}^{\alpha}(v), \mu_{B}^{\alpha}(v) \leq f(v, u), \\
f(v, u), \mu_{B}^{\alpha}(v)>f(v, u),
\end{array}\right.
$$

Since from (3.16) $\mu_{A}(u)+\mu_{B}(v)<1 \Rightarrow f(v, u)<0.25$, but $\mu_{B}^{\alpha}(v) \in[0,1]$, therefore from (3.17) we have

$$
\begin{gathered}
\forall v \in V_{1} \mid \mu_{B}^{\alpha}(v) \wedge f(v, u)=f(v, u)<0.25 \\
\forall v \in V_{2} \mid \mu_{B}^{\alpha}(v) \wedge 1=\mu_{B}^{\alpha}(v)
\end{gathered}
$$

From (3.16)-(3.19) we have

$$
\text { (3.15) }=\left[\int_{U} \bigcup_{v \in V_{2}} \mu_{B}^{\alpha}(v) / u\right]=\int_{U} 1-\mu_{A}^{\alpha}(u) / u=\neg A^{\alpha} \text {. (Q. E. D.). }
$$

For Criteria $I V$-2 let $R\left(D_{1}(y)\right)=\neg B$ then

$$
\begin{aligned}
R\left(D_{2}(x)\right) & =\neg B \circ R\left(D_{1}(y), D_{2}(x)\right) \\
& =\int_{V}\left(1-\mu_{B}(v)\right) / v \circ \int_{V \times U}\left(\left(1-\mu_{B}(v)\right) \rightarrow \mu_{A}(u)\right) /(v, u) \\
& =\int_{U} \bigcup_{v \in V}\left[\left(1-\mu_{B}(v)\right) \wedge\left(\left(1-\mu_{B}(v)\right) \rightarrow \mu_{A}(u)\right)\right] / u
\end{aligned}
$$

From (3.20) and given subsets from (3.14) we have

$$
\begin{aligned}
& R\left(D_{2}(x)\right) \\
= & {\left[\int_{U} \bigcup_{v \in V_{1}}\left[1-\mu_{B}(v) \wedge\left(\mu_{B}(v) \cdot \mu_{A}(u)\right)\right] / u\right] \vee\left[\int_{U} \bigcup_{v \in V_{2}}\left[1-\mu_{B}(v) \wedge 1\right] / u\right] \text { (Q. E. D.) } } \\
= & {\left[\int_{U} \bigcup_{v \in V_{1}}\left[\mu_{A}(u)\right] / u\right] \vee\left[\int_{U} \bigcup_{v \in V_{2}}\left[1-\mu_{B}(v)\right] / u\right]=\int_{U} \mu_{A}(u) / u=A }
\end{aligned}
$$

To illustrate these results we will present couple examples.

\section{Example 1}

Let $U$ and $V$ be two universes of discourses and correspondent fuzzy sets are represented as in (3.6) $A \subset U\left|\mu_{A}: U \rightarrow[0,1], B \subset V\right| \mu_{B}: V \rightarrow[0,1]$; related linguistic scale could consist of the terms like $\{$ “SMALL".., "MEDIUM"..., " $L A R G E$ ' . Let us consider the following cases.

$$
\begin{array}{r}
A \text { labeled "LARGE" } \begin{array}{r}
0.0 / u_{1}+0.1 / u_{2}+0.2 / u_{3}+0.3 / u_{4}+0.4 / u_{5}+0.5 / u_{6} \\
+0.6 / u_{7}+0.7 / u_{8}+0.8 / u_{9}+0.9 / u_{10}+1.0 / u_{11}
\end{array} \\
\begin{aligned}
= & 1.0 / v_{1}+0.9 / v_{2}+0.8 / v_{3}+0.7 / v_{4}+0.6 / v_{5}+0.5 / v_{6} \\
& +0.4 / v_{7}+0.3 / v_{8}+0.2 / v_{9}+0.1 / v_{10}+0.0 / v_{11}
\end{aligned}
\end{array}
$$

The negation of a fuzzy set $B$ would look like

$$
\begin{aligned}
\neg B= & 0.0 / v_{1}+0.1 / v_{2}+0.2 / v_{3}+0.3 / v_{4}+0.4 / v_{5}+0.5 / v_{6} \\
& +0.6 / v_{7}+0.7 / v_{8}+0.8 / v_{9}+0.9 / v_{10}+1.0 / v_{11}
\end{aligned}
$$

The binary relationship matrix $R\left(D_{1}(y), D_{2}(x)\right)$ of a type (3.7) would look like 


\begin{tabular}{cccccccccccc}
\hline$\neg \backslash A$ & $\mathbf{0}$ & $\mathbf{0 . 1}$ & $\mathbf{0 . 2}$ & $\mathbf{0 . 3}$ & $\mathbf{0 . 4}$ & $\mathbf{0 . 5}$ & $\mathbf{0 . 6}$ & $\mathbf{0 . 7}$ & $\mathbf{0 . 8}$ & $\mathbf{0 . 9}$ & $\mathbf{1}$ \\
\hline $\mathbf{0}$ & 1 & 1 & 1 & 1 & 1 & 1 & 1 & 1 & 1 & 1 & 1 \\
$\mathbf{0 . 1}$ & 0 & 1 & 1 & 1 & 1 & 1 & 1 & 1 & 1 & 1 & 1 \\
$\mathbf{0 . 2}$ & 0 & 0.02 & 1 & 1 & 1 & 1 & 1 & 1 & 1 & 1 & 1 \\
$\mathbf{0 . 3}$ & 0 & 0.03 & 0.06 & 1 & 1 & 1 & 1 & 1 & 1 & 1 & 1 \\
$\mathbf{0 . 4}$ & 0 & 0.04 & 0.08 & 0.12 & 1 & 1 & 1 & 1 & 1 & 1 & 1 \\
$\mathbf{0 . 5}$ & 0 & 0.05 & 0.1 & 0.15 & 0.2 & 1 & 1 & 1 & 1 & 1 & 1 \\
$\mathbf{0 . 6}$ & 0 & 0.06 & 0.12 & 0.18 & 0.2 & 0.25 & 1 & 1 & 1 & 1 & 1 \\
$\mathbf{0 . 7}$ & 0 & 0.07 & 0.14 & 0.21 & 0.28 & 0.35 & 0.42 & 1 & 1 & 1 & 1 \\
$\mathbf{0 . 8}$ & 0 & 0.08 & 0.16 & 0.24 & 0.32 & 0.4 & 0.48 & 0.56 & 1 & 1 & 1 \\
$\mathbf{0 . 9}$ & 0 & 0.09 & 0.18 & 0.27 & 0.36 & 0.45 & 0.54 & 0.63 & 0.72 & 1 & 1 \\
$\mathbf{1}$ & 0 & 0.1 & 0.2 & 0.3 & 0.4 & 0.5 & 0.6 & 0.7 & 0.8 & 0.9 & 1 \\
\hline
\end{tabular}

Let $B^{2}$ labeled "very SMALL"

$=1.0 / v_{1}+0.81 / v_{2}+0.64 / v_{3}+0.49 / v_{4}+0.36 / v_{5}+0.25 / v_{6}$

$+0.16 / v_{7}+0.09 / v_{8}+0.04 / v_{9}+0.01 / v_{10}+0.0 / v_{11}$

Applying (3.13)

$$
\begin{aligned}
R\left(D_{2}(x)\right)= & B^{2} \circ R\left(D_{1}(y), D_{2}(x)\right) \\
= & \int_{V} \mu_{B}^{2}(v) / v \circ \int_{V \times U}\left(\left(1-\mu_{B}(v)\right) \rightarrow \mu_{A}(u)\right) /(u, v) \\
= & 1.0 / u_{1}+0.81 / u_{2}+0.64 / u_{3}+0.49 / u_{4}+0.36 / u_{5}+0.25 / u_{6} \\
& +0.16 / u_{7}+0.09 / u_{8}+0.04 / u_{9}+0.01 / u_{10}+0.0 / u_{11} \\
= & \neg A^{2}
\end{aligned}
$$

\section{Example 2}

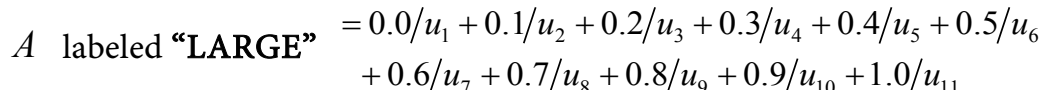

And $B$ also labeled "LARGE"

$$
\begin{aligned}
= & 0.0 / v_{1}+0.1 / v_{2}+0.2 / v_{3}+0.3 / v_{4}+0.4 / v_{5}+0.5 / v_{6} \\
& +0.6 / v_{7}+0.7 / v_{8}+0.8 / v_{9}+0.9 / v_{10}+1.0 / v_{11}
\end{aligned}
$$

The negation of a fuzzy set $B$ would look like

$$
\begin{aligned}
\neg B= & 1.0 / v_{1}+0.9 / v_{2}+0.8 / v_{3}+0.7 / v_{4}+0.6 / v_{5}+0.5 / v_{6} \\
& +0.4 / v_{7}+0.3 / v_{8}+0.2 / v_{9}+0.1 / v_{10}+0.0 / v_{11}
\end{aligned}
$$

The binary relationship matrix $R\left(D_{1}(y), D_{2}(x)\right)$ of a type (3.7) would look like

\begin{tabular}{cccccccccccc}
\hline$\neg B \backslash A$ & $\mathbf{0}$ & $\mathbf{0 . 1}$ & $\mathbf{0 . 2}$ & $\mathbf{0 . 3}$ & $\mathbf{0 . 4}$ & $\mathbf{0 . 5}$ & $\mathbf{0 . 6}$ & $\mathbf{0 . 7}$ & $\mathbf{0 . 8}$ & $\mathbf{0 . 9}$ & $\mathbf{1}$ \\
\hline $\mathbf{1}$ & 0 & 0.1 & 0.2 & 0.3 & 0.4 & 0.5 & 0.6 & 0.7 & 0.8 & 0.9 & 1 \\
$\mathbf{0 . 9}$ & 0 & 0.09 & 0.18 & 0.27 & 0.36 & 0.45 & 0.54 & 0.63 & 0.72 & 1 & 1 \\
$\mathbf{0 . 8}$ & 0 & 0.08 & 0.16 & 0.24 & 0.32 & 0.4 & 0.48 & 0.56 & 1 & 1 & 1 \\
$\mathbf{0 . 7}$ & 0 & 0.07 & 0.14 & 0.21 & 0.28 & 0.35 & 0.42 & 1 & 1 & 1 & 1 \\
$\mathbf{0 . 6}$ & 0 & 0.06 & 0.12 & 0.18 & 0.24 & 0.3 & 1 & 1 & 1 & 1 & 1 \\
\hline
\end{tabular}




\begin{tabular}{|c|c|c|c|c|c|c|c|c|c|c|c|}
\hline \multicolumn{12}{|c|}{ Continued } \\
\hline 0.5 & 0 & 0.05 & 0.1 & 0.15 & 0.2 & 1 & 1 & 1 & 1 & 1 & 1 \\
\hline 0.4 & 0 & 0.04 & 0.08 & 0.12 & 1 & 1 & 1 & 1 & 1 & 1 & 1 \\
\hline 0.3 & 0 & 0.03 & 0.06 & 1 & 1 & 1 & 1 & 1 & 1 & 1 & 1 \\
\hline 0.2 & 0 & 0.02 & 1 & 1 & 1 & 1 & 1 & 1 & 1 & 1 & 1 \\
\hline 0.1 & 0 & 1 & 1 & 1 & 1 & 1 & 1 & 1 & 1 & 1 & 1 \\
\hline 0 & 1 & 1 & 1 & 1 & 1 & 1 & 1 & 1 & 1 & 1 & 1 \\
\hline
\end{tabular}

Applying (3.13)

$$
\begin{aligned}
R\left(D_{2}(x)\right)= & \neg B \circ R\left(D_{1}(y), D_{2}(x)\right) \\
= & \int_{V} 1-\mu_{B}(v) / v \circ \int_{V \times U}\left(\left(1-\mu_{B}(v)\right) \rightarrow \mu_{A}(u)\right) /(u, v) \\
= & 0.0 / u_{1}+0.1 / u_{2}+0.2 / u_{3}+0.3 / u_{4}+0.4 / u_{5}+0.5 / u_{6} \\
& +0.6 / u_{7}+0.7 / u_{8}+0.8 / u_{9}+0.9 / u_{10}+1.0 / u_{11} \\
= & A
\end{aligned}
$$

Let us revisit the fuzzy conditional inference rule (3.5). It will be shown that when the membership function of the observation $\neg B$ is continuous, then the conclusion $A$ depends continuously on the observation; and when the membership function of the relation $R(\neg B, A)$ is continuous then the observation $A$ has a continuous membership function. We start with some definitions. A fuzzy set $A$ with membership function $\mu_{A}: \Re \rightarrow[0,1]=I$, is called a fuzzy number if $A$ is normal, continuous, and convex. The fuzzy numbers represent the continuous possibility distributions of fuzzy terms of the following type

$$
A=\int_{\Re} \mu_{A}(x) / x
$$

Let $A$ be a fuzzy number, then for any $\theta \geq 0$ we define $\varpi_{A}(\theta)$ the modulus of continuity of $A$ by

$$
\varpi_{A}(\theta)=\max _{\left|x_{1}-x_{2}\right| \theta \theta}\left|\mu_{A}\left(x_{1}\right)-\mu_{A}\left(x_{2}\right)\right| .
$$

An $\alpha$-level set of a fuzzy interval $A$ is a non-fuzzy set denoted by $[A]^{\alpha}$ and is defined by $[A]^{\alpha}=\left\{t \in \mathfrak{R} \mid \mu_{A}(t) \geq \alpha\right\}$ for $\alpha \in(0,1]$ and $[A]^{\alpha}=c l\left(\operatorname{supp} \mu_{A}\right)$ for $\alpha=0$. Here we use a metric of the following type

$$
D(A, B)=\sup _{\alpha \in[0,1]} d\left([A]^{\alpha},[B]^{\alpha}\right),
$$

where $d$ denotes the classical Hausdorff metric expressed in the family of compact subsets of $\mathfrak{R}^{2}$, i.e.

$$
d\left([A]^{\alpha},[B]^{\alpha}\right)=\max \left\{\left|a_{1}(\alpha)-b_{1}(\alpha)\right|,\left|a_{2}(\alpha)-b_{2}(\alpha)\right|\right\} .
$$

whereas $[A]^{\alpha}=\left[a_{1}(\alpha), a_{2}(\alpha)\right],[B]^{\alpha}=\left[b_{1}(\alpha), b_{2}(\alpha)\right]$ when the fuzzy sets $A$ and $B$ both have finite support $\left\{x_{1}, \cdots, x_{n}\right\}$ then their Hamming distance is defined as 


$$
H(A, B)=\sum_{i=1}^{n}\left|\mu_{A}\left(x_{i}\right)-\mu_{B}\left(x_{i}\right)\right| .
$$

In the sequel we will use the following lemma.

Lemma 3 [10]

Let $\delta \geq 0$ be a real number and let $A, B$ be fuzzy intervals. If $D(\neg B, A) \leq \delta$, then

$$
\sup _{t \in \mathfrak{R}}\left|\mu_{A}(t)-\mu_{\neg B}(t)\right| \leq \max \left\{\varpi_{A}(\delta), \varpi_{\neg B}(\delta)\right\} .
$$

Consider the fuzzy conditional inference rule (3.5) with different observations $B$ and $B^{1}$ :

$$
\begin{gathered}
\text { Antecedent 1: If } y \text { is } \neg B \text { then } x \text { is } A \\
\text { Antecedent } 2: y \text { is } B \\
\text { Consequent: } x \text { is } \neg A \\
\text { Antecedent } 2: y \text { is } B^{1}
\end{gathered}
$$

According to the fuzzy conditional inference rule (3.5), the membership functions of the conclusions are computed as

$$
\begin{aligned}
& \mu_{\neg A}(u)=\bigcup_{v \in \Re}\left[\mu_{B}(v) \wedge\left(\left(1-\mu_{B}(v)\right) \rightarrow \mu_{A}(u)\right)\right] ; \\
& \mu_{\neg A^{1}}(u)=\bigcup_{v \in \Re}\left[\mu_{B^{1}}(v) \wedge\left(\left(1-\mu_{B}(v)\right) \rightarrow \mu_{A}(u)\right)\right]
\end{aligned}
$$

$\mathrm{Or}$

$$
\begin{aligned}
& 1-\mu_{A}(u)=\sup _{v \in \Re}\left[\mu_{B}(v) \wedge\left(\left(1-\mu_{B}(v)\right) \rightarrow \mu_{A}(u)\right)\right] ; \\
& 1-\mu_{A^{1}}(u)=\sup _{v \in \Re}\left[\mu_{B^{1}}(v) \wedge\left(\left(1-\mu_{B}(v)\right) \rightarrow \mu_{A}(u)\right)\right],
\end{aligned}
$$

The following theorem shows the fact that when the observations are closed to each other in the metric $D($.$) of (3.23) type, then there can be only a small$ deviation in the membership functions of the conclusions.

Theorem 4 (Stability theorem)

Let $\delta \geq 0$ and let $B, B^{1}$ be fuzzy intervals and an implication operation in the fuzzy conditional inference rule (3.5) is from Table S1. If $D\left(B, B^{1}\right) \leq \delta$ then

$$
\sup _{u \in \Re}\left|\mu_{A}(u)-\mu_{A^{1}}(u)\right| \leq \max \left\{\varpi_{\neg B}(\delta), \varpi_{\neg B^{1}}(\delta)\right\}
$$

\section{Proof:}

Given an implication operation in the fuzzy conditional inference rule (3.5) is from Table S1, for the observation $B$ we have

$$
\begin{aligned}
& \exists V_{1}, V_{2} \subset \mathfrak{R}\left|V_{1} \cup V_{2}=V ;\right| V_{1} \cap V_{2}=\varnothing \\
& \Rightarrow \forall v \in V_{1}\left|\mu_{B}(v)<1-\mu_{A}(u) ; \forall v \in V_{2}\right| \mu_{B}(v) \geq 1-\mu_{A}(u) \\
& A=B \circ R\left(D_{1}(y), D_{2}(x)\right) \\
& \quad=\int_{V} \mu_{B}(v) / v \circ \int_{V \times U}\left(\left(1-\mu_{B}(v)\right) \rightarrow \mu_{A}(u)\right) /(v, u) \\
& \quad=\int_{U} \bigcup_{v \in V}\left[\mu_{B}(v) \wedge\left(\left(1-\mu_{B}(v)\right) \rightarrow \mu_{A}(u)\right)\right] / u
\end{aligned}
$$


From (3.13) and given subsets from (3.14) we have

$$
A^{1}=\left[\int_{U} \bigcup_{v \in V_{1}}\left[\mu_{B^{1}}(v) \wedge\left(\mu_{B}(v) \cdot \mu_{A}(u)\right)\right] / u\right] \vee\left[\int_{U} \bigcup_{v \in V_{2}}\left[\mu_{B^{1}}(v) \wedge 1\right] / u\right] .
$$

Then from (3.16) the following is taking place:

$$
\forall v \in V_{1} \mid \mu_{B^{1}}(v) \wedge f(v, u)=\left\{\begin{array}{l}
\mu_{B^{1}}(v), \mu_{B^{1}}(v) \leq f(v, u), \\
f(v, u), \mu_{B^{1}}(v)>f(v, u),
\end{array}\right.
$$

Since from (3.16) $\mu_{A}(u)+\mu_{B}(v)<1 \Rightarrow f(v, u)<0.25$, but $\mu_{B}(v) \in[0,1]$, therefore from (3.17) we have

$$
\begin{gathered}
\forall v \in V_{1} \mid \mu_{B^{1}}(v) \wedge f(v, u)=f(v, u)<0.25 \\
\forall v \in V_{2} \mid \mu_{B^{1}}(v) \wedge 1=\mu_{B^{1}}(v)
\end{gathered}
$$

From (3.27)-(3.29) we have

$$
\text { (3.26) }=\left[\int_{U} \bigcup_{v \in V_{2}} \mu_{B^{1}}(v) / u\right]=\int_{U} 1-\mu_{A^{1}}(u) / u=\neg A^{1}
$$

From (3.28) and (3.29), we see that the difference of the values of conclusions for both $B$ and $B^{1}$ observations for arbitrary fixed $v \in \mathfrak{R}|| \mu_{B}(v)-\mu_{B^{1}}(v) \mid$ is defined as follows

$$
\forall v \in V_{1}|| \mu_{B}(v)-\mu_{B^{1}}(v) \mid=\left\{\begin{array}{l}
\left|\mu_{A}(u)-\mu_{A^{1}}(u)\right|, \\
0,
\end{array}\right.
$$
have

$\forall v \in V_{2}|| \mu_{B}(v)-\mu_{B^{1}}(v)|=| \mu_{A}(u)-\mu_{A^{1}}(u) \mid$. Therefore from Lemma 1 we

$$
\sup _{v \in \Re}\left|\mu_{B}(v)-\mu_{B^{1}}(v)\right|=\sup _{u \in \Re}\left|\mu_{A}(u)-\mu_{A^{1}}(u)\right| \leq \max \left\{\varpi_{\neg B}(\delta), \varpi_{\neg B^{1}}(\delta)\right\}
$$

\section{Theorem 5 (Continuity theorem)}

Let binary relationship $R(v, u)=\left(1-\mu_{B}(v)\right) \rightarrow \mu_{A}(u)$ be continuous. Then $A$ is continuous and $\varpi_{A}(\delta) \leq \varpi_{R}(\delta)$ for each $\delta \geq 0$.

\section{Proof:}

Let $\delta \geq 0$ be a real number and let $u_{1}, u_{2} \in \mathfrak{R}$ such that $\left|u_{1}-u_{2}\right| \leq \delta$. From (3.22) we have $\varpi_{A}(\delta)=\max _{\left|u_{1}-u_{2}\right| \leq \delta}\left|\mu_{A}\left(u_{1}\right)-\mu_{A}\left(u_{2}\right)\right|$. Then

$$
\begin{aligned}
& \left|\mu_{A}\left(u_{1}\right)-\mu_{A}\left(u_{2}\right)\right|=\mid \sup _{v \in \Re}\left[\mu_{B}(v) \wedge\left(\left(1-\mu_{B}(v)\right) \rightarrow \mu_{A}\left(u_{1}\right)\right)\right] \\
& \quad-\sup _{v \in \Re}\left[\mu_{B}(v) \wedge\left(\left(1-\mu_{B}(v)\right) \rightarrow \mu_{A}\left(u_{2}\right)\right)\right] \mid \\
& \leq \sup _{v \in \Re}\left[\mu_{B}(v) \wedge\left|\left(\left(1-\mu_{B}(v)\right) \rightarrow \mu_{A}\left(u_{1}\right)\right)-\left(\left(1-\mu_{B}(v)\right) \rightarrow \mu_{A}\left(u_{2}\right)\right)\right|\right] \\
& \leq \sup _{v \in \Re}\left[\mu_{B}(v) \wedge \varpi_{R}\left(\left|u_{1}-u_{2}\right|\right)\right] \leq \sup _{v \in \Re}\left[\mu_{B}(v) \wedge \varpi_{R}(\delta)\right]=\varpi_{R}(\delta)
\end{aligned}
$$

Results from Theorem 4 and Theorem 5 could be used for formulating another similarity measure, based on Hamming distance between two fuzzy sets 


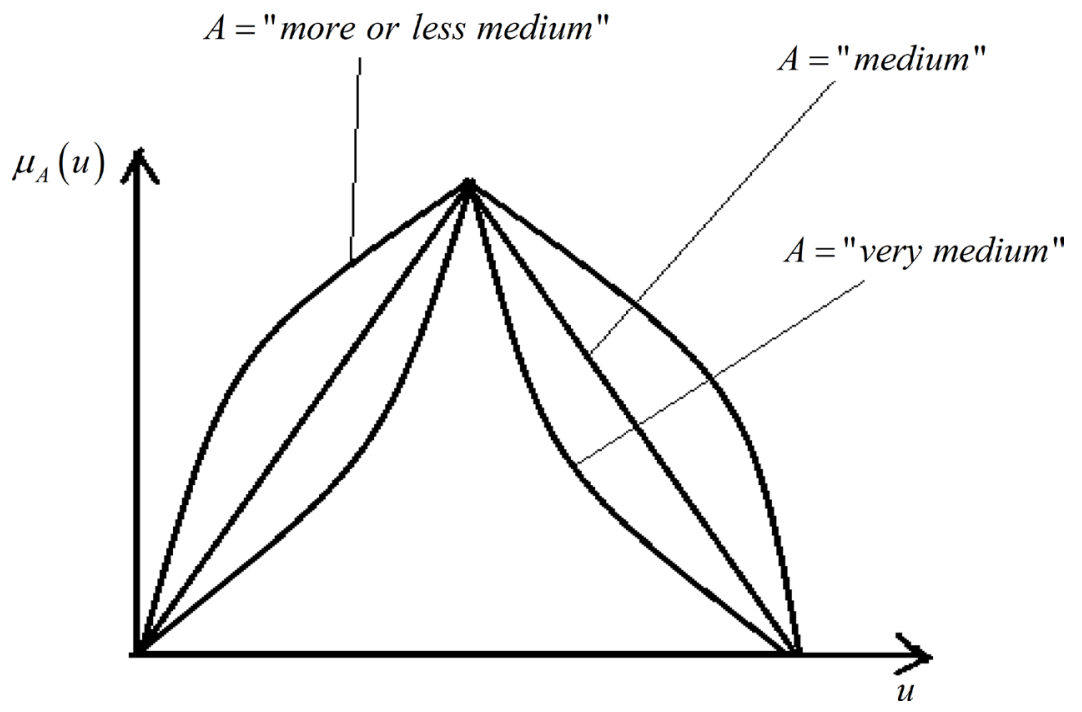

Figure 2. Terms "very" and "more or less".

$A \subseteq U$ and $B \subseteq U$, which presented by non-linear membership functions, i.e.

$$
S I_{1}(A, B)=1-\frac{\sum_{i=1}^{n}\left|\mu_{A}\left(u_{i}\right)-\mu_{B}\left(u_{i}\right)\right|}{n} ; n=\operatorname{Card} U .
$$

For instance let us apply (3.29) to fuzzy sets from Example 1 (see Figure 2):

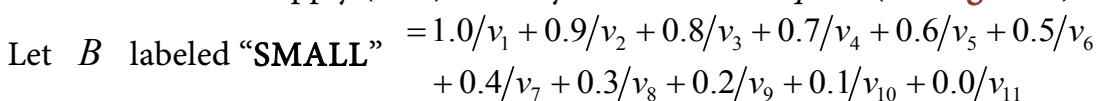

And $B^{2}$ labeled "very SMALL"

$=1.0 / v_{1}+0.81 / v_{2}+0.64 / v_{3}+0.49 / v_{4}+0.36 / v_{5}+0.25 / v_{6}$

$+0.16 / v_{7}+0.09 / v_{8}+0.04 / v_{9}+0.01 / v_{10}+0.0 / v_{11}$

$S I_{1}\left(B, B^{2}\right)=0.85$. It is important to pay attention to a fact that $S I_{1}\left(\neg A, \neg A^{2}\right)=0.85$ from the same Example 1, which confirms results of both Theorem 4 and Theorem 5.

\section{Generalized Modus Tollens Based Inverse Approximate Reasoning}

Let us remind that the scheme for Generalized Modus Tollens (2.3) looks like that

$$
\begin{gathered}
\text { Antecedent 1: If } x \text { is } A \text {, then } y \text { is } B \\
\text { Antecedent 2: } y \text { is } B^{1} \\
\text { Consequent: } x \text { is } A^{1} .
\end{gathered}
$$

First consider classical logic equivalence

$$
a \rightarrow b \equiv \neg b \rightarrow \neg a
$$

The classical logic equivalence (4.2) can be extended in fuzzy logic with implication and negation functions. We use the same fuzzy logic, which operations are presented in Table S1. Let us first proof that (4.2) holds. 
Since

$$
a \rightarrow b=\left\{\begin{array}{l}
(1-a) \cdot b, a>b, \\
1, a \leq b
\end{array}\right.
$$

And because

$$
\neg b \rightarrow \neg a=\left\{\begin{array}{l}
(1-\neg b) \cdot \neg a, \neg b>\neg a, \\
1, \neg b \leq \neg a
\end{array}=\left\{\begin{array}{l}
b \cdot \neg a, a>b, \\
1, a \leq b
\end{array} \equiv \neg a \rightarrow \neg b\right.\right.
$$

Both (4.3) and (4.4) proofs that classical logic equivalence (4.2) holds. It is very important to show that we transform Generalized Modus Tollens rule (4.1) into its equivalent form

$$
\begin{gathered}
\text { Antecedent1: If } y \text { is } \neg B \text { then } x \text { is } \neg A \\
\text { Antecedent } 2: y \text { is } B^{1}
\end{gathered}
$$

Consequent: $\mathrm{x}$ is $A^{1}$.

Let us formulize an inference method for a rule (4.5). Following a standard approaches toward such formalization, let $U$ and $V$ be two universes of discourses and correspondent fuzzy sets be represented as such $A \subset U\left|\mu_{A}: U \rightarrow[0,1], \quad B \subset V\right| \mu_{B}: V \rightarrow[0,1]$.

Whereas given (3.6) a binary relationship for the fuzzy conditional proposition of the type: "If $y$ is $\neg B$ then $x$ is $\neg A$ " for a fuzzy logic is defined as

$$
\begin{aligned}
R\left(D_{1}(y), D_{2}(x)\right) & =\neg B \times V \rightarrow U \times \neg A \\
& =\int_{V \times U}\left(1-\mu_{B}(v)\right) /(v, u) \rightarrow \int_{V \times U}\left(1-\mu_{A}(u)\right) /(v, u) \\
& =\int_{V \times U}\left(\left(1-\mu_{B}(v)\right) \rightarrow\left(1-\mu_{A}(u)\right)\right) /(v, u)
\end{aligned}
$$

Given an implication operator from Table S1 expression (4.6) looks like

$$
\begin{aligned}
& \left(1-\mu_{B}(v)\right) \rightarrow\left(1-\mu_{A}(u)\right) \\
& =\left\{\begin{array}{l}
\mu_{B}(v) \cdot\left(1-\mu_{A}(u)\right), 1-\mu_{B}(v)>1-\mu_{A}(u), \\
1,1-\mu_{B}(v) \leq 1-\mu_{A}(u) .
\end{array}\right. \\
& =\left\{\begin{array}{l}
\mu_{B}(v) \cdot\left(1-\mu_{A}(u)\right), \mu_{A}(u)>\mu_{B}(v), \\
1, \mu_{A}(u) \leq \mu_{B}(v) .
\end{array}\right.
\end{aligned}
$$

And again given a unary relationship $R\left(D_{1}(y)\right)=B^{\prime}$ one can obtain the consequence $R\left(D_{2}(x)\right)$ by applying compositional rule of inference (CRI) to $R\left(D_{1}(y)\right)$ and $R\left(D_{1}(y), D_{2}(x)\right)$ of type (4.6):

$$
\begin{aligned}
R\left(D_{2}(x)\right) & =R\left(D_{1}(y)\right) \circ R\left(D_{1}(y), D_{2}(x)\right) \\
& =\int_{V} \mu_{B^{\prime}}(v) / v \circ \int_{V \times U}\left(\left(1-\mu_{B}(v)\right) \rightarrow\left(1-\mu_{A}(u)\right)\right) /(v, u) \\
& =\int_{U} \bigcup_{v \in V}\left[\mu_{B^{\prime}}(v) \wedge\left(\left(1-\mu_{B}(v)\right) \rightarrow\left(1-\mu_{A}(u)\right)\right)\right] / v
\end{aligned}
$$

In order that Criterion $V$ (see Appendix) is satisfied, that is $R\left(D_{2}(x)\right)=\neg A$ from (3.9) the equality 


$$
\bigcup_{v \in V}\left[\mu_{B}(v) \wedge\left(\left(1-\mu_{B}(v)\right) \rightarrow\left(1-\mu_{A}(u)\right)\right)\right]=\mu_{A}(u)
$$

must be satisfied for arbitrary $u \in U$ and in order that the equality (3.10) is satisfied, it is necessary that the inequality

$$
\mu_{B}(v) \wedge\left(\left(1-\mu_{B}(v)\right) \rightarrow\left(1-\mu_{A}(u)\right)\right) \leq \mu_{A}(u)
$$

holds for arbitrary $u \in U$ and $v \in V$. Let us define new methods of fuzzy conditional inference of the type (4.6), which requires the satisfaction of Criteria $V$-VIII from Appendix.

\section{Theorem 6}

If fuzzy sets $A \subset U\left|\mu_{A}: U \rightarrow[0,1], B \subset V\right| \mu_{B}: V \rightarrow[0,1]$ are defined as (3.6) and $R\left(D_{1}(y), D_{2}(x)\right)$ is defined by (4.6), where

$$
\left(1-\mu_{B}(v)\right) \rightarrow\left(1-\mu_{A}(u)\right)=\left\{\begin{array}{l}
\mu_{B}(v) \cdot\left(1-\mu_{A}(u)\right), \mu_{A}(u)>\mu_{B}(v), \\
1, \mu_{A}(u) \leq \mu_{B}(v) .
\end{array}\right.
$$

then Criteria V,VI,VII and VIII-2 are satisfied.

\section{Proof:}

For Criteria $V$-VII let $R\left(D_{1}(y)\right)=B^{\alpha}(\alpha>0)$ then

$$
\begin{aligned}
& R\left(D_{2}(x)\right)=B^{\alpha} \circ R\left(D_{1}(y), D_{2}(x)\right) \\
&=\int_{V} \mu_{B}^{\alpha}(v) / v \circ \int_{V \times U}\left(\left(1-\mu_{B}(v)\right) \rightarrow\left(1-\mu_{A}(u)\right)\right) /(v, u) \\
&=\int_{U} \bigcup_{v \in V}\left[\mu_{B}^{\alpha}(v) \wedge\left(\left(1-\mu_{B}(v)\right) \rightarrow\left(1-\mu_{A}(u)\right)\right)\right] / u \\
& \exists V_{1}, V_{2} \subset V\left|V_{1} \bigcup V_{2}=V ;\right| V_{1} \cap V_{2}=\varnothing \\
& \Rightarrow \forall v \in V_{1}\left|\mu_{A}(u)>\mu_{B}(v) ; \forall v \in V_{2}\right| \mu_{A}(u) \leq \mu_{B}(v)
\end{aligned}
$$

From (4.11) and given subsets from (4.12) we have

$$
\begin{aligned}
& R\left(D_{2}(x)\right) \\
& =\left[\int_{U} \bigcup_{v \in V_{1}}\left[\mu_{B}^{\alpha}(v) \wedge\left(\mu_{B}(v) \cdot\left(1-\mu_{A}(u)\right)\right] / u\right] \vee\left[\int_{U} \bigcup_{v \in V_{2}}\left[\mu_{B}^{\alpha}(v) \wedge 1\right] / u\right]\right.
\end{aligned}
$$

Let us introduce the following function (as a part of implication operation)

$$
f(v, u)=\mu_{B}(v) \cdot\left(1-\mu_{A}(u)\right) \mid \mu_{A}(u)>\mu_{B}(v) .
$$

Then the following is taking place:

$$
\begin{gathered}
\forall v \in V_{1} \mid \mu_{B}^{\alpha}(v) \wedge f(v, u)=\left\{\begin{array}{l}
\mu_{B}^{\alpha}(v), \mu_{B}^{\alpha}(v) \leq f(v, u), \\
f(v, u), \mu_{B}^{\alpha}(v)>f(v, u),
\end{array}\right. \\
\forall v \in V_{2} \mid \mu_{B}^{\alpha}(v) \wedge 1=\mu_{B}^{\alpha}(v),
\end{gathered}
$$

From (4.15), (4.16) we have

$$
\text { (4.13) }=\left[\int_{U} \bigcup_{v \in V_{2}} \mu_{B}^{\alpha}(v) / u\right]=\int_{U} \mu_{A}^{\alpha}(u) / u=A^{\alpha} \text {. (Q. E. D.). }
$$

For Criteria VIII-2 let $R\left(D_{1}(y)\right)=\neg B$ then 


$$
\begin{aligned}
R\left(D_{2}(x)\right) & =\neg B \circ R\left(D_{1}(y), D_{2}(x)\right) \\
& =\int_{V}\left(1-\mu_{B}(v)\right) / v \circ \int_{V \times U}\left(\left(1-\mu_{B}(v)\right) \rightarrow\left(1-\mu_{A}(u)\right)\right) /(v, u) \\
& =\int_{U} \bigcup_{v \in V}\left[\left(1-\mu_{B}(v)\right) \wedge\left(\left(1-\mu_{B}(v)\right) \rightarrow\left(1-\mu_{A}(u)\right)\right)\right] / u
\end{aligned}
$$

From (4.17) and given subsets from (4.15) and (4.16) we have

$$
R\left(D_{2}(x)\right)=\left[\int_{U} \bigcup_{v \in V_{1}}\left[1-\mu_{B}(v) \wedge f(v, u)\right] / u\right] \vee\left[\int_{U} \bigcup_{v \in V_{2}}\left[1-\mu_{B}(v) \wedge 1\right] / u\right] \text {. Since }
$$

the following is taking place

$$
\left[\left(1-\mu_{B}(v)\right) \wedge f(v, u)\right] \leq\left[\left(1-\mu_{B}(v)\right) \wedge 1\right] .
$$

Therefore

$$
\text { (4.17) }=\left[\int_{U} \bigcup_{v \in V_{2}}\left[\left(1-\mu_{B}(v)\right) \wedge 1\right] / u\right]=\int_{U} 1-\mu_{A}(u) / u=\neg A \text {. (Q. E. D.) }
$$

To illustrate these results we will present couple examples. We use similar fuzzy sets as in Examples 1 and 2.

\section{Example 3}

Let $U$ and $V$ be two universes of discourses and correspondent fuzzy sets are represented as in (3.6) $A \subset U\left|\mu_{A}: U \rightarrow[0,1], B \subset V\right| \mu_{B}: V \rightarrow[0,1]$; related linguistic scale could consist of the terms like \{ $S M A L L " . .$, , "MEDIUM"..., "LARGE" \}. Let us consider the following cases.

$A$ labeled "LARGE" $=0.0 / u_{1}+0.1 / u_{2}+0.2 / u_{3}+0.3 / u_{4}+0.4 / u_{5}+0.5 / u_{6}$ $+0.6 / u_{7}+0.7 / u_{8}+0.8 / u_{9}+0.9 / u_{10}+1.0 / u_{11}$

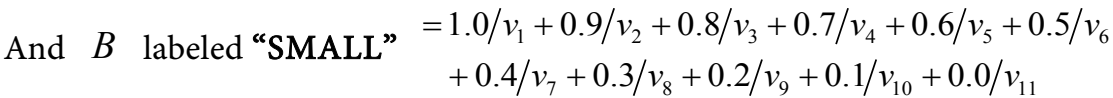

The negations of fuzzy sets $A \quad B$ would look like

$$
\begin{aligned}
\neg A= & 1.0 / u_{1}+0.9 / u_{2}+0.8 / u_{3}+0.7 / u_{4}+0.6 / u_{5}+0.5 / u_{6} \\
& +0.4 / u_{7}+0.3 / u_{8}+0.2 / u_{9}+0.1 / u_{10}+0.0 / u_{11} \\
\neg B= & 0.0 / v_{1}+0.1 / v_{2}+0.2 / v_{3}+0.3 / v_{4}+0.4 / v_{5}+0.5 / v_{6} \\
& +0.6 / v_{7}+0.7 / v_{8}+0.8 / v_{9}+0.9 / v_{10}+1.0 / v_{11}
\end{aligned}
$$

The binary relationship matrix $R\left(D_{1}(y), D_{2}(x)\right)$ of a type (4.6) would look like

\begin{tabular}{cccccccccccc}
\hline$\neg B \backslash \neg A$ & 1 & 0.9 & 0.8 & 0.7 & 0.6 & 0.5 & 0.4 & 0.3 & 0.2 & 0.1 & 0 \\
\hline $\mathbf{0}$ & 1 & 1 & 1 & 1 & 1 & 1 & 1 & 1 & 1 & 1 & 1 \\
$\mathbf{0 . 1}$ & 0.9 & 1 & 1 & 1 & 1 & 1 & 1 & 1 & 1 & 1 & 1 \\
$\mathbf{0 . 2}$ & 0.8 & 0.72 & 1 & 1 & 1 & 1 & 1 & 1 & 1 & 1 & 1 \\
$\mathbf{0 . 3}$ & 0.7 & 0.63 & 0.56 & 1 & 1 & 1 & 1 & 1 & 1 & 1 & 1 \\
$\mathbf{0 . 4}$ & 0.6 & 0.54 & 0.48 & 0.42 & 1 & 1 & 1 & 1 & 1 & 1 & 1 \\
$\mathbf{0 . 5}$ & 0.5 & 0.45 & 0.4 & 0.35 & 0.3 & 1 & 1 & 1 & 1 & 1 & 1 \\
$\mathbf{0 . 6}$ & 0.4 & 0.36 & 0.32 & 0.28 & 0.24 & 0.2 & 1 & 1 & 1 & 1 & 1 \\
\hline
\end{tabular}




\section{Continued}

\begin{tabular}{cccccccccccc}
\hline $\mathbf{0 . 7}$ & 0.3 & 0.27 & 0.24 & 0.21 & 0.18 & 0.15 & 0.12 & 1 & 1 & 1 & 1 \\
$\mathbf{0 . 8}$ & 0.2 & 0.18 & 0.16 & 0.14 & 0.12 & 0.1 & 0.08 & 0.06 & 1 & 1 & 1 \\
$\mathbf{0 . 9}$ & 0.1 & 0.09 & 0.08 & 0.07 & 0.06 & 0.05 & 0.04 & 0.03 & 0.02 & 1 & 1 \\
$\mathbf{1}$ & 0 & 0 & 0 & 0 & 0 & 0 & 0 & 0 & 0 & 0 & 1 \\
\hline
\end{tabular}

Let $B^{2}$ labeled "very SMALL"

$=1.0 / v_{1}+0.81 / v_{2}+0.64 / v_{3}+0.49 / v_{4}+0.36 / v_{5}+0.25 / v_{6}$

$+0.16 / v_{7}+0.09 / v_{8}+0.04 / v_{9}+0.01 / v_{10}+0.0 / v_{11}$

Applying (4.7)

$$
\begin{aligned}
& R\left(D_{2}(x)\right)=B^{2} \circ R\left(D_{1}(y), D_{2}(x)\right) \\
= & \int_{V} \mu_{B}^{2}(v) / v \circ \int_{V \times U}\left(\left(1-\mu_{B}(v)\right) \rightarrow\left(1-\mu_{A}(u)\right)\right) /(v, u) \\
= & 0.0 / u_{1}+0.01 / u_{2}+0.04 / u_{3}+0.09 / u_{4}+0.16 / u_{5}+0.25 / u_{6} \\
& +0.36 / u_{7}+0.49 / u_{8}+0.64 / u_{9}+0.81 / u_{10}+1.0 / u_{11} \\
= & A^{2}
\end{aligned}
$$

("very LARGE").

\section{Example 4}

$A$ labeled "LARGE" $\begin{aligned}= & 0.0 / u_{1}+0.1 / u_{2}+0.2 / u_{3}+0.3 / u_{4}+0.4 / u_{5}+0.5 / u_{6} \\ & +0.6 / u_{7}+0.7 / u_{8}+0.8 / u_{9}+0.9 / u_{10}+1.0 / u_{11}\end{aligned}$

And $B$ also labeled "LARGE"

$$
\begin{aligned}
= & 0.0 / v_{1}+0.1 / v_{2}+0.2 / v_{3}+0.3 / v_{4}+0.4 / v_{5}+0.5 / v_{6} \\
& +0.6 / v_{7}+0.7 / v_{8}+0.8 / v_{9}+0.9 / v_{10}+1.0 / v_{11}
\end{aligned}
$$

The negations of fuzzy sets $A \quad B$ would look like

$$
\begin{aligned}
\neg A= & 1.0 / u_{1}+0.9 / u_{2}+0.8 / u_{3}+0.7 / u_{4}+0.6 / u_{5}+0.5 / u_{6} \\
& +0.4 / u_{7}+0.3 / u_{8}+0.2 / u_{9}+0.1 / u_{10}+0.0 / u_{11} \\
\neg B= & 1.0 / v_{1}+0.9 / v_{2}+0.8 / v_{3}+0.7 / v_{4}+0.6 / v_{5}+0.5 / v_{6} \\
& +0.4 / v_{7}+0.3 / v_{8}+0.2 / v_{9}+0.1 / v_{10}+0.0 / v_{11}
\end{aligned}
$$

The binary relationship matrix $R\left(D_{1}(y), D_{2}(x)\right)$ of a type (4.6) would look like

\begin{tabular}{cccccccccccc}
\hline$\neg B \backslash \neg A$ & 1 & 0.9 & $\mathbf{0 . 8}$ & $\mathbf{0 . 7}$ & $\mathbf{0 . 6}$ & $\mathbf{0 . 5}$ & $\mathbf{0 . 4}$ & $\mathbf{0 . 3}$ & $\mathbf{0 . 2}$ & $\mathbf{0 . 1}$ & $\mathbf{0}$ \\
\hline $\mathbf{1}$ & 1 & 0 & 0 & 0 & 0 & 0 & 0 & 0 & 0 & 0 & 0 \\
$\mathbf{0 . 9}$ & 1 & 1 & 0.08 & 0.07 & 0.06 & 0.05 & 0.04 & 0.03 & 0.02 & 0.01 & 0 \\
$\mathbf{0 . 8}$ & 1 & 1 & 1 & 0.14 & 0.12 & 0.1 & 0.08 & 0.06 & 0.04 & 0.02 & 0 \\
$\mathbf{0 . 7}$ & 1 & 1 & 1 & 1 & 0.18 & 0.15 & 0.12 & 0.09 & 0.06 & 0.03 & 0 \\
$\mathbf{0 . 6}$ & 1 & 1 & 1 & 1 & 1 & 0.2 & 0.16 & 0.12 & 0.08 & 0.04 & 0 \\
$\mathbf{0 . 5}$ & 1 & 1 & 1 & 1 & 1 & 1 & 0.2 & 0.15 & 0.1 & 0.05 & 0 \\
$\mathbf{0 . 4}$ & 1 & 1 & 1 & 1 & 1 & 1 & 1 & 0.18 & 0.12 & 0.06 & 0 \\
$\mathbf{0 . 3}$ & 1 & 1 & 1 & 1 & 1 & 1 & 1 & 1 & 0.14 & 0.07 & 0 \\
$\mathbf{0 . 2}$ & 1 & 1 & 1 & 1 & 1 & 1 & 1 & 1 & 1 & 0.08 & 0 \\
$\mathbf{0 . 1}$ & 1 & 1 & 1 & 1 & 1 & 1 & 1 & 1 & 1 & 1 & 0 \\
$\mathbf{0}$ & 1 & 1 & 1 & 1 & 1 & 1 & 1 & 1 & 1 & 1 & 1 \\
\hline
\end{tabular}


Applying (4.7)

$$
\begin{aligned}
R\left(D_{2}(x)\right)= & \neg B \circ R\left(D_{1}(y), D_{2}(x)\right) \\
= & \int_{V} 1-\mu_{B}(v) / v \circ \int_{V \times U}\left(\left(1-\mu_{B}(v)\right) \rightarrow\left(1-\mu_{A}(u)\right)\right) /(v, u) \\
= & 1.0 / u_{1}+0.9 / u_{2}+0.8 / u_{3}+0.7 / u_{4}+0.6 / u_{5}+0.5 / u_{6} \\
& +0.4 / u_{7}+0.3 / u_{8}+0.2 / u_{9}+0.1 / u_{10}+0.0 / u_{11} \\
= & \neg A
\end{aligned}
$$

\section{Concluding Remarks}

In this article, we presented a systemic approach toward a fuzzy logic based formalization of an approximate reasoning methodology in a fuzzy resolution. We derived a truth value of $A$ from both values of $B \rightarrow A$ and $B$ by some mechanism. We used a t-norm fuzzy logic, in which an implication operator is a root of both graduated conjunction and disjunction operators. We investigated features of correspondent fuzzy resolvent, which was based on introduced operators. We proposed two types of Similarity Measures for both linear and non-linear membership functions. We applied this approach to both generalized modus-ponens/modus-tollens syllogisms, for which we formulated a set of Criterion. The content of this investigation is well-illustrated with artificial examples.

\section{References}

[1] Tserkovny, A. (2017) A t-Norm Fuzzy Logic for Approximate Reasoning. Journal of Software Engineering and Applications, 10, 639-662. https://doi.org/10.4236/jsea.2017.107035

[2] Robinson, J.A. (1965) A Machine Oriented Logic Based on the Resolution Principle. Journal of the ACM, 12, 23-41. https://doi.org/10.1145/321250.321253

[3] Mukaidono, M. (1988) Fuzzy Inference of Resolution Style. In: Yager, R.R., Ed., Fuzzy Set and Possibility Theory, Pergamon Press, New York, 224-231.

[4] Mondal, B. and Raha, S. (2013) Approximate Reasoning in Fuzzy Resolution. International Journal of Intelligence Science, 3, 86-98. https://doi.org/10.4236/ijis.2013.32010

[5] Aliev, R.A. and Tserkovny, A. (1988) The Knowledge Representation in Intelligent Robots Based on Fuzzy Sets. Soviet Mathematics Doklady, 37, 541-544.

[6] Aliev, R.A., Mamedova, G.A. and Tserkovny, A.E. (1991) Fuzzy Control Systems, Energoatomizdat, Moscow.

[7] Aliev, R.A., Fazlollahi, B. and Aliev, R.R., Eds. (2004) Soft Computing and Its Application in Business and Economics. In: Studies in Fuzziness and Soft Computing, Vol. 157, Physica-Verlag, A Spriger-Verlag Company, Berlin Heidelberg. https://doi.org/10.1007/978-3-540-44429-9

[8] Aliev, R.A. and Tserkovny, A. (2011) Systemic Approach to Fuzzy Logic Formalization for Approximate Reasoning. Information Sciences, 181, 1045-1059. https://doi.org/10.1016/j.ins.2010.11.021

[9] Tserkovny, A. (2016) Fuzzy Logic for Incidence Geometry. The Scientific World Journal, 2016, Article ID: 9057263. https://doi.org/10.1155/2016/9057263

[10] Fuller, R. and Zimmermann, H.-J. (1993) On Zadeh's Compositional Rule of Infe- 
rence. In: Lowen, R. and Roubens, M., Eds., Fuzzy Logic. State of the Art, Theory and Decision Library, Series D, Kluwer Academic Publisher, Dordrecht, 193-200. https://doi.org/10.1007/978-94-011-2014-2_19 


\section{Appendix}

Table S1. A Fuzzy Logic Operations.

\begin{tabular}{|c|c|c|}
\hline Name & Designation & Value \\
\hline Tautology & $A^{\mathrm{I}}$ & 1 \\
\hline Controversy & $A^{\mathrm{O}}$ & 0 \\
\hline Negation & $\neg A$ & $1-A$ \\
\hline Disjunction & $A \vee B$ & $\left\{\begin{array}{l}a \cdot b, a+b<1 \\
1, a+b \geq 1\end{array}\right.$ \\
\hline Conjunction & $A \wedge B$ & $\left\{\begin{array}{l}a \cdot b, a+b>1 \\
0, a+b \leq 1\end{array}\right.$ \\
\hline Implication & $A \rightarrow B$ & $\left\{\begin{array}{l}(1-a) \cdot b, a>b \\
1, a \leq b\end{array}\right.$ \\
\hline Equivalence & $A \leftrightarrow B$ & $\left\{\begin{array}{l}(1-b) \cdot a, a<b \\
1, a=b \\
(1-a) \cdot b, a>b\end{array}\right.$ \\
\hline Pierce Arrow & $A \downarrow B$ & $\left\{\begin{array}{l}(1-a) \cdot(1-b), a+b<1, \\
0, a+b \geq 1\end{array}\right.$ \\
\hline Shaffer Stroke & $A \uparrow B$ & $\left\{\begin{array}{l}(1-a) \cdot(1-b), a+b>1, \\
1, a+b \leq 1\end{array}\right.$ \\
\hline
\end{tabular}

Table S2. A Fuzzy Logic Implication Operation.

\begin{tabular}{cccccccccccc}
\hline$a \rightarrow b$ & 0 & 0.1 & 0.2 & 0.3 & 0.4 & 0.5 & 0.6 & 0.7 & 0.8 & 0.9 & 1 \\
\hline $\mathbf{0}$ & 1 & 1 & 1 & 1 & 1 & 1 & 1 & 1 & 1 & 1 & 1 \\
$\mathbf{0 . 1}$ & 0 & 1 & 1 & 1 & 1 & 1 & 1 & 1 & 1 & 1 & 1 \\
$\mathbf{0 . 2}$ & 0 & 0.08 & 1 & 1 & 1 & 1 & 1 & 1 & 1 & 1 & 1 \\
$\mathbf{0 . 3}$ & 0 & 0.07 & 0.14 & 1 & 1 & 1 & 1 & 1 & 1 & 1 & 1 \\
$\mathbf{0 . 4}$ & 0 & 0.06 & 0.12 & 0.18 & 1 & 1 & 1 & 1 & 1 & 1 & 1 \\
$\mathbf{0 . 5}$ & 0 & 0.05 & 0.1 & 0.15 & 0.2 & 1 & 1 & 1 & 1 & 1 & 1 \\
$\mathbf{0 . 6}$ & 0 & 0.04 & 0.08 & 0.12 & 0.16 & 0.2 & 1 & 1 & 1 & 1 & 1 \\
$\mathbf{0 . 7}$ & 0 & 0.03 & 0.06 & 0.09 & 0.12 & 0.15 & 0.18 & 1 & 1 & 1 & 1 \\
$\mathbf{0 . 8}$ & 0 & 0.02 & 0.04 & 0.06 & 0.08 & 0.1 & 0.12 & 0.14 & 1 & 1 & 1 \\
$\mathbf{0 . 9}$ & 0 & 0.01 & 0.02 & 0.03 & 0.04 & 0.05 & 0.06 & 0.07 & 0.08 & 1 & 1 \\
$\mathbf{1}$ & 0 & 0 & 0 & 0 & 0 & 0 & 0 & 0 & 0 & 0 & 1 \\
\hline
\end{tabular}

\section{Criterion I}

Antecedent 1: If $y$ is $\neg B$ then $x$ is $A$ Antecedent 2: $y$ is $B$

Consequent: $\mathrm{x}$ is $\neg A$. 
Criterion II

Antecedent 1: If $y$ is $\neg B$ then $x$ is $A$ Antecedent 2: $y$ is very $B$

Consequent: $x$ is $\neg($ very $A)$.

Criterion III

Antecedent 1: If $y$ is $\neg B$ then $x$ is $A$ Antecedent 2: $y$ is more or less $B$

Consequent: $x$ is $\neg($ more or less $A)$.

Criterion IV-1

Antecedent 1: If $y$ is $\neg B$ then $x$ is $A$ Antecedent 2: $y$ is $\neg B$

Consequent: $x$ is unknown

Criterion IV-2

Antecedent 1: If $y$ is $\neg B$ then $x$ is $A$ Antecedent 2: $y$ is $\neg B$

Consequent: $x$ is $A$.

\section{Criterion V}

Antecedent 1: If $y$ is $\neg B$ then $x$ is $\neg A$

Antecedent 2: $y$ is $B$

\section{Consequent: $\mathrm{x}$ is $A$.}

\section{Criterion VI}

Antecedent 1: If $y$ is $\neg B$ then $x$ is $\neg A$ Antecedent 2: $y$ is very $B$

Consequent: $x$ is very $A$

\section{Criterion VII}

Antecedent 1: If $y$ is $\neg B$ then $x$ is $\neg A$

Antecedent 2: $y$ is more or less $B$

Consequent: $\mathrm{x}$ is more or less $A$

\section{Criterion VIII-1}

Antecedent 1: If $y$ is $\neg B$ then $x$ is $\neg A$

Antecedent 2: $y$ is $\neg B$

Consequent: $x$ is unknown 


\section{Criterion VIII-2}

Antecedent 1: If $y$ is $\neg B$ then $x$ is $\neg A$

Antecedent 2: $y$ is $\neg B$

Consequent: $x$ is $\neg A$.

Submit or recommend next manuscript to SCIRP and we will provide best service for you:

Accepting pre-submission inquiries through Email, Facebook, LinkedIn, Twitter, etc. A wide selection of journals (inclusive of 9 subjects, more than 200 journals)

Providing 24-hour high-quality service

User-friendly online submission system

Fair and swift peer-review system

Efficient typesetting and proofreading procedure

Display of the result of downloads and visits, as well as the number of cited articles Maximum dissemination of your research work

Submit your manuscript at: http://papersubmission.scirp.org/

Or contact jsea@scirp.org 